\title{
THE ZERO DISPERSION LIMIT OF THE KORTEWEG-DE VRIES EQUATION WITH PERIODIC INITIAL DATA
}

\author{
STEPHANOS VENAKIDES
}

ABstract. We study the initial value problem for the Korteweg-de Vries equation

$$
u_{t}-6 u u_{x}+\varepsilon^{2} u_{x x x}=0
$$

in the limit of small dispersion, i.e., $\varepsilon \rightarrow 0$.

When the unperturbed equation

$$
u_{t}-6 u u_{x}=0
$$

develops a shock, rapid oscillations arise in the solution of the perturbed equation (i) In our study:

a. We compute the weak limit of the solution of (i) for periodic initial data as $\varepsilon \rightarrow 0$.

b. We show that in the neighborhood of a point $(x, t)$ the solution $u(x, t, \varepsilon)$ can be approximated either by a constant or by a periodic or by a quasiperiodic solution of equation (i). In the latter case the associated wavenumbers and frequencies are of order $O(1 / \varepsilon)$.

c. We compute the number of phases and the wave parameters associated with each phase of the approximating solution as functions of $x$ and $t$.

d. We explain the mechanism of the generation of oscillatory phases.

Our computations in a and $c$ are subject to the solution of the Lax-Levermore evolution equations (7.7). Our results in $b-d$ rest on a plausible averaging assumption.

\section{Introduction.}

Description of the problem. We study the initial value problem for the Korteweg-de Vries (KdV) equation

$$
\begin{gathered}
u_{t}-6 u u_{x}+\varepsilon^{2} u_{x x x}=0, \\
u(x, 0, \varepsilon)=-v(x)
\end{gathered}
$$

in the limit $\varepsilon \rightarrow 0$. In this limit equation (0.1a) is a model for the formation and propagation of dispersive shocks in one space dimension. We examine the problem with periodic initial data of period $p$. We normalize the initial data to satisfy

$$
\max _{x \in \mathbf{R}} v(x)=1, \quad \min _{x \in \mathbf{R}} v(x)=0 .
$$

Received by the editors July 31, 1985 and, in revised form, April 24, 1986.

1980 Mathematics Subject Classification (1985 Revision). Primary 35Q20; Secondary 35P20, 35R30.

Research supported by the Office of Naval Research, the Air Force Office of Scientific Research, the Army Research Office, and the National Science Foundation. 
The physical and mathematical mechanisms of weak dispersion are dramatically different from the better known ones of weak dissipation. For example, in the weak dissipation limit of the Burger's equation $u_{t}-6 u u_{x}-\varepsilon^{2} u_{x x}=0$, the limit of the solution $u(x, t, \varepsilon)$ exists in a strong sense as $\varepsilon \rightarrow 0$. The limit of the solution displays shock discontinuities whose speed and stability are fixed by the Rankine-Hugoniot jump condition and the entropy condition. This is to be contrasted with the small dispersion limit of the $\mathrm{KdV}$ equation (0.1) in which a shock emits rapid oscillations which spread into the medium. As a result the limit of the solution $u(x, t, \varepsilon)$ of $(0.1)$ as $\varepsilon \rightarrow 0$ exists only weakly in the shock region.

History of the problem. The initial value problem $(0.1 \mathrm{a}, \mathrm{b})$ has been studied by Lax and Levermore [6] for special initial data, which decay as $x \rightarrow \pm \infty$. The problem was later studied for other types of decaying initial data by Venakides $[11,12]$ and by Levermore [21]. The main quantity computed in [6] and [11] is the weak limit of the solution $u(x, t, \varepsilon)$ as $\varepsilon \rightarrow 0$. More specifically, $u(x, t, \varepsilon)$ is represented as the second derivative in $x$, of a quantity which converges uniformly in compact sets of $(x, t)$ as $\varepsilon \rightarrow 0$. The method employed in these cases is the inverse scattering transformation. The generation of oscillations studied in [12] can be considered as a limiting case of the present study.

Our main results. a. We compute the weak limit of the solution of $(0.1)$ for periodic initial data as $\varepsilon \rightarrow 0$. The limit is weak in the sense that $\int^{x} u\left(x^{\prime}, t, \varepsilon\right) d x^{\prime}$ converges uniformly in compact sets of $(x, t)$.

b. We show that in the neighborhood of a point $(x, t)$ the solution $u(x, t, \varepsilon)$ can be approximated either by a constant or by a periodic or by a quasiperiodic solution of equation ( $0.1 \mathrm{a})$ with wavenumbers and frequencies of order $O(1 / \varepsilon)$.

c. We compute the number of phases and the wave parameters associated with each phase of the approximating solution as functions of $x$ and $t$.

d. We explain the mechanism of the generation of oscillatory phases.

Our computations in a and $\mathrm{c}$ are subject to the solution of the Lax-Levermore evolution equations (7.7). Our results in $b-d$ rest on a plausible averaging assumption.

Interpretation of our results on the generation of oscillations. In this discussion we assume $x$ and $t$ to be parameters which have taken on some fixed value. The solution of $(0.1)$ near $(x, t)$ is given in terms of the stretched variables $y$ and $\tau$ by $u(x+\varepsilon y, t+\varepsilon \tau, \varepsilon)$. It satisfies the $\mathrm{KdV}$ equation

$$
u_{\tau}-6 u u_{y}+u_{y y y}=0 .
$$

We show in $\S 8$ that when $\varepsilon$ is sufficiently small, we can approximate $u(x+\varepsilon y, t+$ $\varepsilon \tau, \varepsilon)$ either by a constant or by a periodic or by a quasiperiodic soliton solution of (0.4). We denote this by $U(y, \tau ; x, t) . U(y, \tau ; x, t)$ approximates $u(x+\varepsilon y, t+\varepsilon \tau, \varepsilon)$ almost uniformly in $(y, \tau)$. Its wave parameters, with the exception of phase shifts, are independent of $\varepsilon$ (hence our suppression of $\varepsilon$ in the notation). According to the theory of the $\mathrm{KdV}$ equation the wave parameters of $U(y, \tau ; x, t)$ (excluding phase shifts) and the position of the baseline are uniquely determined by the spectrum of the selfadjoint operator on the line $-\infty<y<\infty$ :

$$
\mathscr{L}_{(x, t)}=d^{2} / d y^{2}-U(y, \tau ; x, t) .
$$


The spectrum of this operator depends on $x$ and $t$ and is independent of $\varepsilon$. It is also independent of $\tau$. The number of gaps in the spectrum of $\mathscr{L}_{(x, t)}$ equals the number of interacting oscillatory phases present in $U(y, \tau ; x, t)$. The dependence of the spectral endpoints on $x$ and $t$ gives the modulation of the local wave parameters.

We define the family of sets

$$
\Sigma_{t}=\left\{(x, \xi): \xi \text { is an element of the spectrum of } \mathscr{L}_{(x, t)}\right\} .
$$

Clearly knowledge of the sets $\Sigma_{t}$ is equivalent to knowledge of the waveform (up to phase shifts) at each $(x, t)$ in the limit $\varepsilon \rightarrow 0$.

We denote the boundary of the set $\Sigma_{t}$ in $\mathbf{R}^{2}$ by $g_{t}$. The boundary $g_{t}$ is a natural object to study. At $t=0 g_{t}$ is simply the graph of the curve $\xi=v(x)$ of the initial data of (0.1). Indeed, at $t=0$

$$
\lim _{\varepsilon \rightarrow 0} u(x+\varepsilon y, 0+\varepsilon \tau, \varepsilon)=u(x, 0)=-v(x) .
$$

The approximating solution $U(y, \tau ; x, 0)$ to $(0.2)$ is the "constant" $-v(x)$, i.e., a zero-phase solution. The associated operator is given by

$$
\mathscr{L}_{(x, 0)}=d^{2} / d y^{2}+v(x)
$$

with spectrum $(-\infty, v(x))$. When $t>0$ and before breaktime (i.e., at times at which no two characteristics of the equation

$$
u_{t}-6 u u_{x}=0
$$

intersect, the strong limit of $u(x, t, \varepsilon)$ exists as $\varepsilon \rightarrow 0$ and equals $u(x, t, 0)$. As in the case $t=0, g_{t}$ is the graph of the function $\xi=-u(x, t, 0)$. We let

$$
\beta_{0}^{2}(x, t)=-u(x, t, 0)=-\lim _{\varepsilon \rightarrow 0} u(x+\varepsilon y, t+\varepsilon \tau, \varepsilon)
$$

for all $t$ before breaktime.

After breaktime, when the limit of $u(x, t, \varepsilon)$ ceases to exist in a strong sense in the shock region and $u(x+\varepsilon y, t+\varepsilon \tau, \varepsilon)$ is a modulated periodic or quasiperiodic solution in the variables $y, \tau$, we show that the evolution of $g_{t}$ is governed by equations (7.7) first derived by Lax and Levermore [6]. The curve $g_{t}$ becomes the graph of a multivalued function of $x$. This is not the multivalued function obtained by naively taking the values carried by all characteristics of (0.6) arriving at a point $(x, t)$. While multivaluedness is not acceptable for the solution of $(0.6)$, it is perfectly admissible for the curve $g_{t}$ which has been defined as the boundary of a set in $\mathbf{R}^{2}$. Multivaluedness in this case signifies that the spectrum of the associated operator $\mathscr{L}_{(x, t)}$ develops gaps. To each of these corresponds a phase of oscillation. In $\S 7$ these gaps are denoted by $\left(\beta_{3}^{2}, \beta_{2}^{2}\right),\left(\beta_{5}, \beta_{4}^{2}\right)$, etc.; the maximum of the spectrum by $\beta_{0}^{2}$. The $\beta_{i}$ 's are seen to satisfy the modulation equations derived by Whitham [13] for a single phase wave and by Flaschka, Forest, and McLaughlin [4] in the multiphase case. These equations are not sufficient to capture the evolution of the curve $g_{t}$ globally, because they cannot describe the generation of a new phase created when the modulation equations of the existing phases develop a shock. On the other hand the Lax-Levermore evolution equations do not display a similar problem in describing the evolution of $g_{t}$. The singular problem (0.1) has thus been transformed to the regular, in principle well-posed, initial value problem, the Lax-Levermore evolution 
equations (7.7). We do not yet have a rigorous proof for the existence (local or global) and uniqueness of the solution of the Lax-Levermore evolution equations beyond breaktime.

Description of our method. Following the works of Dubrovin, Matveev, and Novikov [3] and McKean, van Moerbeke, and Trubowitz [7, 8, 9] we represent the solution $u(x, t, \varepsilon)$ of the $\mathrm{KdV}$ equation in terms of the spectral data of the associated selfadjoint Hill's operator $\mathscr{L}_{\varepsilon}(t)=\varepsilon^{2} d^{2} / d s^{2}-u(s, t, \varepsilon)$ on the line $-\infty<s<\infty$. As $\varepsilon \rightarrow 0$, we obtain a representation of the form

$$
\begin{gathered}
u(x, t, \varepsilon)=-2 \frac{\partial}{\partial x} \varepsilon \sum_{i=1}^{\infty} f\left(v_{i}\right)+o(1), \\
\sum_{i=1}^{\infty} \varepsilon b_{j}\left(\nu_{i}, \varepsilon\right)=a_{j}(x, t, \varepsilon)+o(1), \quad j=1,2, \ldots,
\end{gathered}
$$

where $a_{j}, b_{j}$, and $f$ are known functions, and $\left(\nu_{i}(x, t, \varepsilon)\right)_{i=1}$ are eigenvalues of the operator $\mathscr{L}_{\varepsilon}(t)$ in the interval $[x, x+p]$ with Dirichlet boundary data. We show that the system of equations ( 0.9$)$ tends to a continuum limit as $\varepsilon \rightarrow 0$. This is an integral equation, for a certain density function, and is solvable by the Wiener-Hopf method used by Lax and Levermore [6, II]. We refer to it as the basic integral equation. In the same limit, equation (0.8) yields the weak limit of $u(x, t, \varepsilon)$ as a functional of the density function which solves the basic integral equation.

When $\varepsilon$ is nonzero but sufficiently small, we employ the solution of the basic integral equation to recover information on the solution to the discrete equations $(0.8)$ and $(0.9)$. This allows us to discern the mechanism by which oscillations arise in $u(x, t, \varepsilon)$.

Our method differs from the method employed by Lax and Levermore [6] and by Venakides [11] for the problem on the whole line. In [6] the reflection coefficient is proved negligible and the exact solution is represented in terms of the $N$-soliton formula. In [11] the exact solution is again approximated by what essentially amounts to a continuum of interacting solitons. In both of these cases the limit $\varepsilon \rightarrow 0$ is taken on the exact solution represented by the integral of a real exponential function over a complicated measure.

Advantages of the method. Our method:

(a) Solves the periodic problem.

(b) Obtains the weak limit of $u(x, t, \varepsilon)$ as a first derivative in the variable $x$ of a quantity converging in the strong sense.

(c) When $\varepsilon$ is small but nonzero, it describes basic features of the waveform in the shock region.

(d) We conjecture that the method can be used to solve other integrable systems in the continuum limit, provided they have a representation

$$
\begin{gathered}
u(x, t, \varepsilon)=F\left(\nu_{1}, \nu_{2}, \ldots ; x, t, \varepsilon\right), \\
G_{j}\left(\nu_{1}, \ldots ; x, t, \varepsilon\right)=0, \quad j=1,2, \ldots,
\end{gathered}
$$

where equations (0.11) should be interpreted as a complete set of first integrals. Interesting candidates are the nonlinear Schrödinger and sine-Gordon equations. Applying our method to these equations-especially in the case in which, unlike the 
$\mathrm{KdV}$ equation, the associated operator is not selfadjoint-should not necessarily yield results similar to ours, and presumably requires a nontrivial extension of our work. This is confirmed by a look at existing results. For example, Forest and McLaughlin [15] and Ercolani, Forest, and McLaughlin [17] show that unlike the $\mathrm{KdV}$ wavetrains many sine-Gordon wavetrains have nonhyperbolic modulation equations. These wavetrains are modulationally unstable and should not be expected to evolve from nonoscillatory initial data.

Related topics. a. This study gives an analytical proof of a mechanism by which a coherent finite-dimensional spatial structure passes through a shock by restabilizing into another, slightly larger, finite-dimensional structure.

b. Given our detailed derivation of the oscillations, the Young measure at each space-time point may be computed. This computation is carried out by McLaughlin [14]. Thus, we are in a case where the questions raised by the compensated compactness approach of Tartar, DiPerna, and others, regarding the generation and propagation of oscillations in conservation laws can be answered more or less explicitly.

c. Our study can be considered a special case of the more general problem of the KdV-Burger equation $u_{t}-6 u u_{x}+\varepsilon^{2} u_{x x x}=\delta u_{x x}$, which displays the simultaneous presence of weak dissipation and dispersion. In this case the dissipative (resp. dispersive) term dominates when $\delta$ and $\varepsilon$ tend to zero satisfying $\varepsilon^{2} \ll \delta$ (resp. $\delta \ll \varepsilon^{2}$ ) (see Schonbek [18]). On the boundary $\delta / \varepsilon^{2}=O(1)$ the results of Forest and McLaughlin [16] apply.

Organization. In $\S 1$ we write the solution to the periodic $\mathrm{KdV}$ equation in the implicit form of $(0.8)-(0.9)$ and in $\$ 2$ we discuss the general effect of $\varepsilon \rightarrow 0$. In $\S \S 3-6$ the limit $\varepsilon \rightarrow 0$ is taken. The basic integral equation is derived, and the weak limit is obtained as a functional of the solution of this equation. In $\$ 7$ we outline the solution of the basic integral equation using results of Lax and Levermore. Finally in $\S 8$ we outline the mechanism of the emergence of oscillations. We give a more detailed account of the generation of oscillations in [12], where the case of decaying initial data has been examined.

1. Representation of periodic solutions. A p-periodic solution of the Korteweg-de Vries equation

$$
u_{t}-6 u u_{x}+u_{x x x}=0
$$

defined on the real line and satisfying mild regularity assumptions has the representation $[2,9]$

$$
u(x, t)=-\xi_{0}+2 \sum_{i=1}^{g}\left[\nu_{i}(x, t)-\frac{\xi_{2 i-1}+\xi_{2 i}}{2}\right],
$$

where $\left[\xi_{2 i-2}, \xi_{2 i-1}\right]$ is the $i$ th spectral band of the selfadjoint Hill's operator $\mathscr{L}(t)=d^{2} / d s^{2}-u(s, t)$ on the real line $-\infty<s<\infty$, and $\nu_{i}(x, t)$ is the unique solution of the eigenvalue problem $\mathscr{L}(t) \psi_{i}(s)=\nu_{i}(x, t) \psi_{i}(s), \psi_{i}(x)=\psi_{i}(x+p)$ $=0$ which satisfies $\xi_{2 i-1} \leqslant \nu_{i} \leqslant \xi_{2 i}$. We call the $\nu_{i}$ 's the Dirichlet spectrum. We have $\xi_{0}>\xi_{1} \geqslant \nu_{1} \geqslant \xi_{2}>\xi_{3} \geqslant \nu_{2} \geqslant \xi_{4}>\xi_{5} \geqslant \nu_{3} \geqslant \cdots$. The set of $\xi_{i}$ 's is also characterized as the union of the simple spectra of $\mathscr{L}(t)$ over the interval $[x, x+p]$ 
with (a) periodic $(\psi(x)=\psi(x+p))$, (b) antiperiodic $(\psi(x)=-\psi(x+p))$ boundary conditions. The number $g$ of spectral gaps can be finite or infinite. As $x$ and $t$ vary, the $\xi_{i}$ 's remain constant, and $\nu_{j}(x, t)$ oscillates in the $j$ th spectral gap $\left[\xi_{2 j}, \xi_{2 j-1}\right]$ according to the equations

$$
\frac{\partial \nu_{j}}{\partial x}=\frac{2 \pi^{2} j^{2}}{p^{2}} \frac{R\left(\nu_{j}\right)^{1 / 2}}{W_{j}\left(\nu_{1} ; \nu_{2}, \ldots\right)}
$$

$$
\frac{\partial \nu_{j}}{\partial t}=2\left\{u(x, t)-2 \nu_{j}\right\} \frac{\partial \nu_{j}}{\partial x}=\frac{4 \pi^{2} j^{2}}{p^{2}}\left\{u(x, t)-2 \nu_{j}\right\} \frac{R\left(\nu_{j}\right)^{1 / 2}}{W_{j}\left(\nu_{1}, \ldots, \nu_{g}\right)},
$$

where for $j=1,2, \ldots, g$ :

$$
\begin{gathered}
R\left(\nu_{j}\right)=\left(\nu_{j}-\xi_{0}\right) \prod_{i=1}^{g} \frac{\left(\nu_{j}-\xi_{2 i-1}\right)\left(\nu_{j}-\xi_{2 i}\right)}{\pi^{4} i^{4} p^{-4}}, \\
W_{j}\left(\nu_{1}, \nu_{2}, \ldots\right)=\prod_{\substack{i=1 \\
i \neq j}}^{g} \frac{\left(\nu_{j}-\nu_{i}\right)}{\pi^{2} i^{2} p^{-2}} .
\end{gathered}
$$

These products are well defined when $g=\infty$ because

$$
\xi_{2 i} \sim-\pi^{2} i^{2} / p^{2} \text { as } i \rightarrow \infty,
$$

width of $i$ th gap $=\xi_{2 i-1}-\xi_{2 i}$ decays fast as $i \rightarrow \infty$,

This decay is exponential if $u(x, t)$ is analytic in $x$. The sign of the square root $R\left(\nu_{j}\right)^{1 / 2}$ changes every time $\nu_{j}$ reaches an endpoint of the interval $\left[\xi_{2 j}, \xi_{2 j-1}\right]$. $R\left(\nu_{j}\right)^{1 / 2}$ can be made single-valued, if it is defined to take positive and negative values respectively on two different copies of the interval $\left[\xi_{2 j}, \xi_{2 j-1}\right]$. The corresponding endpoints of the two copies are then identified and topologically $\nu_{j}$ varies on a circle.

Our $\nu_{j}$ 's and $\xi_{i}$ 's are the $-\mu_{j}$ 's and $-\lambda_{i}$ 's respectively of the traditional notation of this representation.

In the following theorem, we integrate explicitly the system of differential equations (1.3). This system was derived by Trubowitz [9] in the case of infinitely many gaps. Our theorem is a generalization to infinitely many gaps of the integration of the system in the finite case as given in [2].

THEOREM 1.1. Let

$$
P(\nu)=\prod_{i=1}^{g} \frac{\nu-\rho_{i}}{\pi^{2} i^{2} p^{-2}}, \quad P_{j}(\nu)=\frac{P(\nu)}{\nu \cdot-\rho_{j}},
$$

where $\left(\rho_{i}\right)_{i=1}^{g}$ are real numbers satisfying

$$
-\xi_{0}+2 \sum_{i=1}^{g}\left(\rho_{i}-\frac{\xi_{2 i}+\xi_{2 i-1}}{2}\right)=0
$$

$$
\rho_{i} \in\left[\xi_{2 i}, \xi_{2 i+1}\right]=\text { ith gap for all but finitely many } i \text { 's. }
$$


Then

(1.11)

$$
u(x, t)=-\xi_{0}+2 \sum_{i=1}^{g}\left\{\nu_{i}(x, t)-\frac{\xi_{2 i}+\xi_{2 i-1}}{2}\right\}=\frac{\partial}{\partial x} \sum_{i=1}^{g} \int^{\nu_{i}(x, t)} \frac{P(\nu)}{R(\nu)^{1 / 2}} d \nu
$$

and

$$
\sum_{i=0}^{g} \int_{\nu_{i}\left(x_{0}, t_{0}\right)}^{\nu_{i}(x, t)} \frac{1}{2} \frac{P_{j}(\nu)}{R(\nu)^{1 / 2}} d \nu=x-x_{0}-4 \rho_{j}\left(t-t_{0}\right), \quad j=1,2,3, \ldots
$$

The theorem holds for $g$ finite or $g=\infty$.

REMARK 1.2. The fact that topologically $\nu_{i}$ varies in a circle means that the value of the integral in (1.12) corresponding to the index $i$ depends on how many times $\nu_{i}$ winds around the $i$ th circle as $x$ and $t$ vary. The winding number is independent of the path connecting points $\left(x_{0}, t_{0}\right)$ and $(x, t)$ on the $x-t$ plane.

The proof of the theorem is given in Appendix I.

2. The effect of the small parameter $\varepsilon$. The problem $(0.1 \mathrm{a}-\mathrm{b})$ is transformed by the rescaling $x^{\prime}=x / \varepsilon, t^{\prime}=t / \varepsilon$ into

$$
u_{t^{\prime}}-6 u u_{x^{\prime}}+u_{x^{\prime} x^{\prime} x^{\prime}}=0, \quad u\left(x^{\prime}, 0\right)=-v\left(\varepsilon x^{\prime}\right) .
$$

Representation (1.11)-(1.12) gives

$$
u(x, t, \varepsilon)=-\xi_{0}+2 \sum_{i=1}^{g}\left(\nu_{i}(x, t)-\frac{\xi_{2 i}+\xi_{2 i-1}}{2}\right)=\frac{\partial}{\partial x} \sum_{i=1}^{g} \varepsilon \int^{\nu_{i}(x, t)} \frac{P(\nu)}{R(\nu)^{1 / 2}} d \nu .
$$

The $\nu_{i}(x, t)$ are constrained by the inequalities $\xi_{2 i} \leqslant \nu_{i}(x, t) \leqslant \xi_{2 i-1}$ and satisfy the equation

$$
\sum_{i=1}^{g} \varepsilon \int_{\nu_{i}\left(x_{0}, t_{0}\right)}^{\nu_{i}(x, t)} \frac{1}{2} \frac{P_{j}(\nu)}{R(\nu)^{1 / 2}} d \nu=x-x_{0}-4 \rho_{j}\left(t-t_{0}\right), \quad j=1,2, \ldots
$$

The $\left(\xi_{i}\right)_{i=0}^{2 g}$ are the simple periodic/antiperiodic eigenvalues and $\left(\nu_{i}(x, t)\right)_{i=1}^{g}$ are the corresponding Dirichlet eigenvalues of the selfadjoint operator $\mathscr{L}_{\varepsilon}(t)=\varepsilon^{2} d^{2} / d s^{2}-$ $u(s, t)$ in the interval $[x, x+p]$. At $t=0$ we have $\mathscr{L}_{\varepsilon}(0)=\varepsilon^{2} d^{2} / d s^{2}+v(s)$.

The $\xi_{i}$ 's and $\nu_{i}\left(x_{0}, 0\right)$ 's are computed in Appendix III by the W.K.B. method as $\varepsilon \rightarrow 0$. In this limit, the width of the spectral gaps decays exponentially in $\varepsilon$, in the region of negative eigenvalue parameter $\xi$. We show later that the gaps of this region may be neglected. Thus, the significant Dirichlet eigenvalues satisfy $\nu_{i} \geqslant 0$. In view of the inequality $\xi_{0}>\xi_{1} \geqslant \nu_{1} \geqslant \xi_{2}>\xi_{3} \geqslant \nu_{2} \geqslant \xi_{4}>\xi_{5} \geqslant \nu_{3} \geqslant \cdots$, the significant Dirichlet eigenvalues are given by the truncated sequence $\nu_{1}, \nu_{2}, \ldots, \nu_{N(\varepsilon)}$, where the index $N(\varepsilon)$ is conveniently defined by the equality

$$
\xi_{2 N}=0 \text {. }
$$

The assumption that there is some $N$ satisfying (2.4) can be made without loss of generality. 
In region $0<\xi<1$ of the eigenvalue parameter $\xi$, the width of the spectral bands decays exponentially as $\varepsilon \rightarrow 0$. The distance between two successive bands is generically of order $\varepsilon$. In the region $\xi>1$ there is no spectrum. It follows that $N(\varepsilon)=O(1 / \varepsilon)$ as $\varepsilon \rightarrow 0$. Our problem will thus be reduced to one with $N(\varepsilon)=$ $O(1 / \varepsilon)$ degrees of freedom. This observation, which we prove rigorously, corresponds to the Ansatz in Lax and Levermore [6] that the reflection coefficient can be neglected. In fact, if we let the period of the initial data tend to infinity, the $N(\varepsilon)$ exponentially thin bands in the region $\xi>0$ tend to become points: the discrete eigenvalues of Lax-Levermore. The exponentially small gaps in the region $\xi<0$ correspond to the negligible contribution from the reflection coefficient.

The functions $P$ and $P_{j}$ of representation (2.2)-(2.3) depend on the $\rho_{i}$ 's of Theorem 1.1. The following selection of the $\rho_{i}$ 's will simplify the representation

$$
\rho_{i}=\left\{\begin{array}{l}
\frac{1}{2}\left(\xi_{2 i-2}+\xi_{2 i-1}\right)=\text { midpoint of } i \text { th band when } i \leqslant N(\varepsilon), \\
\frac{1}{2}\left(\xi_{2 i}+\xi_{2 i-1}\right)=\text { midpoint of } i \text { th gap when } i>N(\varepsilon) .
\end{array}\right.
$$

We observe that the $\rho_{i}$ 's defined by (2.5) satisfy both constraints (1.10).

3. The representation equations. We start the calculation by computing the integrals in (2.2)-(2.3). First we define

$(3.1 \mathrm{a}-\mathrm{b}) \quad \sigma(\nu)=-\operatorname{sgn} \frac{P(\nu)}{R(\nu)^{1 / 2}}= \pm 1 \quad$ and $\quad \sigma_{i}(x, t, \varepsilon)=\sigma\left(\nu_{i}(x, t, \varepsilon)\right)$.

$\sigma(\nu)$ depends critically on the sign of the square root of $R(\nu)$. Relations (1.2)-(1.4) and (1.9) imply

$$
\sigma_{i}=-\operatorname{sgn} \frac{P\left(\nu_{i}\right)}{R\left(\nu_{i}\right)^{1 / 2}}=\operatorname{sgn} \frac{\partial \nu_{i}}{\partial x}=-\operatorname{sgn} \frac{\partial \nu_{i}}{\partial t} .
$$

We define the following indefinite integrals in which the variable $\nu$ ranges over the gap region:

$$
\begin{aligned}
& \int^{\nu} \frac{P(\nu)}{R(\nu)^{1 / 2}} d \nu=A(\nu) \sigma(\nu), \\
& \int^{\nu} \frac{P_{j}(\nu)}{R(\nu)^{1 / 2}} d \nu=D_{j}(\nu) \sigma(\nu) .
\end{aligned}
$$

The constant of integration in each gap is defined conveniently by the relations

$$
A(0)=0 \quad\left(\text { recall } \xi_{2 N}=0\right),
$$

$$
\begin{gathered}
A\left(\xi_{2 i-2}\right)=A\left(\xi_{2 i-1}\right) \quad \text { when } i \leqslant N, \\
A\left(\rho_{i}\right)=0 \text { when } i>N .
\end{gathered}
$$

Similarly,

$$
\begin{gathered}
D_{j}(0)=0, \\
D_{j}\left(\xi_{2 i-2}\right)=D_{j}\left(\xi_{2 i-1}\right) \quad \text { when } i \leqslant N, \\
D_{j}\left(\rho_{i}\right)=0 \quad \text { when } i>N .
\end{gathered}
$$


Formula (2.2) yields

$$
\int^{x} u(s, t, \varepsilon) d s=\sum_{i=1}^{g} \varepsilon A\left(\nu_{i}\right) \sigma_{i}(x, t, \varepsilon)+\text { const },
$$

where "constant" means independent of $x$. The integration of (2.3) needs some attention, because of the winding numbers in the integrals. We define $m_{k}(x, t, \varepsilon)$ to be the number of times that one of the $\nu_{i}$ 's (necessarily a unique one) takes the value $\xi_{k}$, as the space-time variables vary from $\left(x_{0}, t_{0}\right)$ to $(x, t)$. We define $m_{0}(x, t, \varepsilon)=0$. We obtain the formula

$$
\int_{\nu_{i}\left(x_{0}, t_{0}, \varepsilon\right)}^{\nu_{i}(x, t, \varepsilon)} \frac{P_{j}(\nu)}{R(\nu)^{1 / 2}} d \nu=\left.D_{j}(\nu) \sigma(\nu)\right|_{\nu_{i}\left(x_{0}, t_{0}, \varepsilon\right)} ^{\nu_{i}(x, t, \varepsilon)}+2\left[m_{2 i-1} D_{j}\left(\xi_{2 i-1}\right)-m_{2 i} D_{j}\left(\xi_{2 i}\right)\right] .
$$

We substitute (3.4) in (2.3). After rearranging some terms and using (3.2d) we obtain

$$
\begin{aligned}
& \left.\sum_{i=1}^{g} \varepsilon D_{j}\left(\nu_{i}\right) \sigma_{i}\right|_{\nu_{i}=\nu_{i}\left(x_{0}, t_{0}, \varepsilon\right)} ^{\nu_{i}(x, t, \varepsilon)}-2 \varepsilon \sum_{i=1}^{N}\left[m_{2 i-1} D_{j}\left(\xi_{2 i-1}\right)-m_{2 i} D_{j}\left(\xi_{2 i}\right)\right] \\
& -2 \varepsilon \sum_{i=N+1}^{g}\left[m_{2 i-1} D_{j}\left(\xi_{2 i-1}\right)-m_{2 i} D_{j}\left(\xi_{2 i}\right)\right]=x-x_{0}-4 \rho_{j}\left(t-t_{0}\right),
\end{aligned}
$$

where $j=1,2, \ldots, g$. The calculation of the functions $A(\nu)$ and $D_{j}(\nu)$ is performed in Appendix II. We find that an error of order $o(1)$ as $\varepsilon \rightarrow 0$, will appear in (3.3), if $A(\nu)$ is replaced by

$$
\bar{A}(\nu)= \begin{cases}-2 \nu^{1 / 2} & \text { when } \nu>0 \\ 0 & \text { when } \nu<0\end{cases}
$$

Equation (3.6) implies that the influence of the negative $\nu_{i}$ 's (i.e., when $i>N$ ) on (3.3) is negligible as $\varepsilon \rightarrow 0$. This in turn means that we need consider only the equations corresponding to $j \leqslant N$ in (3.5). Again we find that an error of $o(1)$ as $\varepsilon \rightarrow 0$ will appear in (3.5) if $D_{j}(\nu)$ is replaced by

$$
\bar{D}_{j}(\nu)= \begin{cases}-\frac{1}{\eta_{j}} \log \left|\frac{\nu^{1 / 2}-\eta_{j}}{\nu^{1 / 2}+\eta_{j}}\right| & \text { when }\left|\nu-\eta_{j}^{2}\right|>\varepsilon^{2}, \\ -\frac{1}{\eta_{j}} \log \left(\left|\nu-\eta_{j}^{2}\right|+\left[\left(\nu-\eta_{j}^{2}\right)^{2}-\delta_{j}^{2}\right]^{1 / 2}\right) & \text { when } \delta_{j} \leqslant\left|\nu-\eta_{j}^{2}\right|<\varepsilon^{2}, \\ 0 & \text { when } \nu<0,\end{cases}
$$

where, for $j=1,2, \ldots N(\varepsilon)$,

$$
\eta_{j}^{2}=\rho_{j}=\text { center of } j \text { th spectral band, }
$$

and the square roots have positive sign. 
We substitute (3.6) in (3.3) and (3.7) in (3.5). By making some simple manipulations and using $\nu_{i} \sim \eta_{i}$ we obtain

THEOREM 3.1.

$$
\begin{aligned}
& \int_{\substack{i=1 \\
i \neq j \\
i \neq j+1}}^{x} u(s, t, \varepsilon) d s=-2 \sum_{i=1}^{N(\varepsilon)} \varepsilon \eta_{i} \sigma_{i}(x, t, \varepsilon)+o(1) \quad(\varepsilon \rightarrow 0), \\
& \quad+r_{j}(x, t, \varepsilon)-r_{j}\left(x_{0}, t_{0}, \varepsilon\right)+n_{j}(x, t, \varepsilon) \gamma_{j} \\
& =\eta_{j}\left(x-x_{0}\right)-4 \eta_{j}^{3}\left(t-t_{0}\right) \\
& \quad+\sum_{\substack{i=1 \\
i \neq j}}^{N(\varepsilon)} \varepsilon\left[\frac{1}{2}\left(1-\sigma_{i}(x, t, \varepsilon)\right)-n_{i}(x, t, \varepsilon)\right] \log \left|\frac{\eta_{i}-\eta_{j}}{\eta_{i}+\eta_{j}}\right| \\
& \left.\left.\left.\quad x_{0}, t_{0}, \varepsilon\right)\right)-n_{i}\left(x_{0}, t_{0}, \varepsilon\right)\right] \log \left|\frac{\eta_{i}-\eta_{j}}{\eta_{i}+\eta_{j}}\right|+o(1),
\end{aligned}
$$

where $j=1,2, \ldots N(\varepsilon)$,

$$
\begin{gathered}
n_{j}(x, t, \varepsilon)=m_{2 j-1}(x, t, \varepsilon)-m_{2 j-2}(x, t, \varepsilon)=\text { integer }, \\
\gamma_{j}=-\varepsilon \log \delta_{j}, \\
r_{j}(x, t, \varepsilon)=\frac{\varepsilon}{2}\left\{\eta_{j} \bar{D}_{j}\left(\nu_{j}\right) \sigma_{j}+\eta_{j-1} \bar{D}_{j}\left(\nu_{j-1}\right) \sigma_{j-1}\right\} .
\end{gathered}
$$

We remark that, since the band widths $\delta_{i}$ given by equation (III 38) of Appendix III are exponentially small in $\varepsilon$, the quantities $\gamma_{j}=-\varepsilon \log \delta_{j}$ have a first order contribution in (3.10). For the same reason the quantities $r_{j}$ defined by (3.13) may also have a first order contribution in (3.10). The $n_{j}$ 's correspond to the differences of the winding numbers in neighboring gaps. Whereas the winding numbers, in general, tend to infinity as $\varepsilon \rightarrow 0$, it will be seen that their differences are independent of $\varepsilon$ in this limit.

TheOREM 3.2. As $\varepsilon \rightarrow 0$ it is true that

$$
\begin{gathered}
m_{j}(x, t, \varepsilon)=O\left(\left(x-x_{0}\right) / \varepsilon\right) \quad \text { uniformly in } j . \\
n_{j}(x, t, \varepsilon)=O\left(x-x_{0}\right) \quad \text { uniformly in } j . \\
\left|r_{j}(x, t, \varepsilon)\right| \leqslant \frac{1}{2} \gamma_{j}+o(1) .
\end{gathered}
$$

Before proving Theorem 3.2 we outline some of the results of our analysis of the initial data in Appendix III which we will use in the proof. Theorem 3.2 is essentially valid for general periodic initial data. However, for reasons of simplicity our analysis is restricted to initial data having a single well per period. In this case we show in Figure 3.1 the graphs of $\nu_{j}(x, 0, \varepsilon)$ and $\nu_{j-1}(x, 0, \varepsilon)$ when $j=j(\varepsilon)$ is such that $\eta_{j(\varepsilon)} \rightarrow \eta \in(0,1)$ as $\varepsilon \rightarrow 0$. 


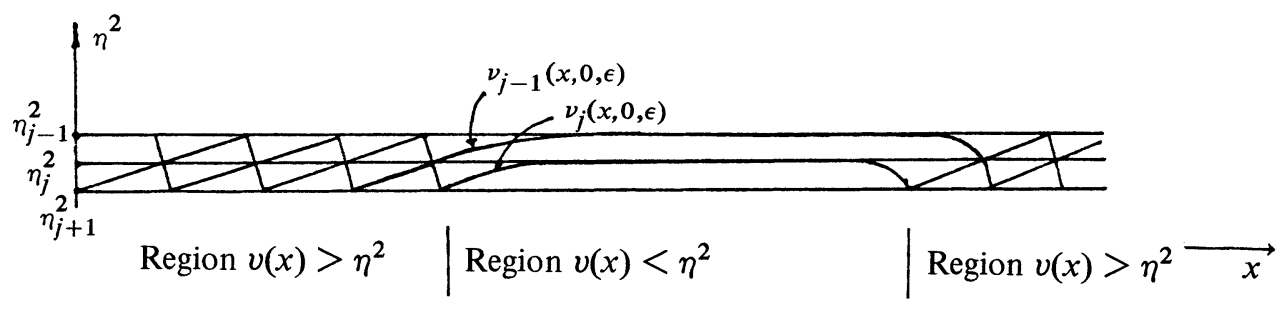

Figure 3.1

The following basic facts pictured in Figure 3.1 are proven in Appendix III:

(a) The distances $\eta_{j}^{2}-\eta_{j+1}^{2}$ and $\eta_{j-1}^{2}-\eta_{j}^{2}$ are of order $\varepsilon$. The width of the $j$ th band decays exponentially as $\varepsilon \rightarrow 0$. These results are proven in Theorem III.4.

(b) Region $\eta^{2}>v(x)$. In this region $\nu_{j}(x, 0, \varepsilon)$ equals an endpoint of the $j$ th gap (specifically the point $\eta_{j}^{2}-\delta_{j}$ ) at exactly one $x$ in each period. The distance $\left|\nu_{j}-\eta_{j}^{2}\right|$ decays exponentially as $\varepsilon \rightarrow 0$ (see Theorem III.5).

(c) Region $\eta^{2}<v(x)$. The graph of $\nu_{j}(x, 0, \varepsilon)$ is triangular with slopes of order 1 (see Theorem III.5). By (a) above, in the region $\left\{x: \eta^{2}<v(x)\right\}, \nu_{j}(x, 0, \varepsilon)$ equals an endpoint of the $j$ th gap at a number of $x$ 's per period which is of order $1 / \varepsilon$. Furthermore,

$$
\nu_{j-1}(\tilde{x}, 0, \varepsilon)=\eta_{j}^{2}+\delta_{j} \Leftrightarrow \nu_{j}(\tilde{x}, 0, \varepsilon)=\eta_{j}^{2}-\delta_{j} .
$$

Thus, the contribution of this region to $n_{j}(x, 0)$ in $(3.11)$ is zero.

Claim 3.3. If $\tilde{x}_{0}$ satisfies (3.17) then there is a continuous function $\tilde{x}(t)$ which satisfies

$$
\begin{gathered}
\nu_{j-1}(\tilde{x}(t), t, \varepsilon)=\eta_{j}^{2}+\delta_{j}, \\
\nu_{j}(\tilde{x}(t), t, \varepsilon)=\eta_{j}^{2}-\delta_{j}, \\
\tilde{x}(0)=\tilde{x}_{0} .
\end{gathered}
$$

REMARK 3.4. For reasons of simplicity we have allowed a slight inaccuracy in equations (3.17)-(3.18). Instead of $\tilde{x}$ we should have $\tilde{x}$ in (3.18a) and $\hat{x}$ in (3.18b) with $|\tilde{x}(t)-\hat{x}(t)|$ decaying exponentially as $\varepsilon \rightarrow 0$. Avoiding this inaccuracy would add annoying complication and nothing of substance to our calculation.

REMARK 3.5. Claim 3.3 states that the topology of Figure 3.1 is preserved for $t>0$.

REMARK 3.6. In the region $\eta^{2}<v(x), \nu_{j}(x, 0, \varepsilon)$ performs a spatial oscillation with frequency of order $O(1 / \varepsilon)$. Such fast oscillations are thus present even at $t=0$ but their contribution to (2.2) cancel out. This will no longer be true beyond breaktime. The mechanism by which total cancellation ceases and an overall fast oscillation emerges is explained in $\S 8$.

Proof of Claim 3.3. We take the ratios

$$
\frac{\partial \nu_{j+1}}{\partial x} / \frac{\partial \nu_{j}}{\partial x} \text { and } \frac{\partial \nu_{j-1}}{\partial t} / \frac{\partial \nu_{i}}{\partial t}
$$


in equations (1.3)-(1.4). As long as

$$
\left|\nu_{j}-\nu_{j-1}\right| \ll \varepsilon
$$

which is true at $x=\tilde{x}, t=0$ we can cancel most of the factors in the ratios. We obtain

$$
\frac{d \nu_{j-1}}{d \nu_{j}} \sim \frac{\sigma_{j-1}\left[\left(\nu_{j-1}-\eta_{j}^{2}+\delta_{j}\right)\left(\nu_{j-1}-\eta^{2}-\delta_{j}\right)\right]^{1 / 2}}{\sigma_{j}\left[\left(\nu_{j}-\eta_{j}^{2}+\delta_{j}\right)\left(\nu_{j}-\eta_{j}^{2}-\delta_{j}\right)\right]^{1 / 2}} .
$$

This integrates to

$$
\begin{aligned}
\log \{ & \left.\nu_{j-1}-\eta_{j}^{2}+\sigma_{j-1}\left[\left(\nu_{j-1}-\eta_{j}^{2}\right)^{2}-\delta_{j}^{2}\right]^{1 / 2}\right\} \\
& \sim \log \left\{\eta_{j}^{2}-\nu_{j}-\sigma_{j}\left[\left(\eta_{j}^{2}-\nu_{j}\right)^{2}-\delta_{j}^{2}\right]^{1 / 2}\right\}+\text { const. }
\end{aligned}
$$

Allowing for the inaccuracy we mentioned in Remark 3.4 we replace " " with $"="$. At $t=0, x=\tilde{x}$ we have

$$
\nu_{j-1}-\eta_{j}^{2}=\eta_{j}^{2}-\nu_{j}=\delta_{j} .
$$

Thus in (3.21) the constant is zero. There exists a unique $\tilde{x}(t)$ satisfying $(3.18 \mathrm{~b}-\mathrm{c})$. By (3.21) $\tilde{x}(t)$ satisfies (3.18a) as well. Q.E.D.

Proof of TheOREM 3.2. Let $p$ be the period of the initial data. By points (c) in our earlier discussion of initial data, $m_{j}(x+p, 0, \varepsilon)-m_{j}(x, 0, \varepsilon)=O(1 / \varepsilon)$ uniformly in $j$. The integers $m_{j}(x+p, t, \varepsilon)-m_{j}(x, t, \varepsilon)$, where $\varepsilon>0$ is fixed, are topological invariants independent of $x$ and $t$. Relation (3.14) follows. Relation (3.15) is true at $t=0$ by points (a) and (b) above. It is true at $t>0$ because the topology of Figure 3.1 is preserved according to Claim 3.3.

We now prove (3.16). We have from (3.13)

$$
\begin{aligned}
r_{j}(x, t, \varepsilon)= & -(\varepsilon / 2) \sigma_{j-1} \log \left\{\nu_{j-1}-\eta_{j}^{2}+\left[\left(\nu_{j-1}-\eta_{j}^{2}\right)^{2}-\delta_{j}^{2}\right]^{1 / 2}\right\} \\
& -(\varepsilon / 2) \sigma_{j} \log \left\{\nu_{j}-\eta_{j}^{2}+\left[\left(\nu_{j}-\eta_{j}^{2}\right)^{2}-\delta_{j}^{2}\right]^{1 / 2}\right\} .
\end{aligned}
$$

Each term in the right-hand side of (3.23) is absolutely bounded by $\frac{1}{2} \gamma_{j}$. Furthermore $\left|\nu_{i}-\eta_{j}^{2}\right|>\varepsilon^{2}$ where $i=j$ or $i=j-1 \Rightarrow$ corresponding term in the right-hand side of (3.23) is $o(1)$ as $\varepsilon \rightarrow 0$. Thus the only case in which (3.16) could be violated is at some $(x, t, \varepsilon)$ satisfying simultaneously

(3.24a, b, c) $\quad \sigma_{j}=\sigma_{j-1}, \quad\left|\nu_{j}-\eta_{j}^{2}\right|<\varepsilon^{2}$, and $\quad\left|\nu_{j-1}-\eta_{j}^{2}\right|<\varepsilon^{2}$.

One can easily verify that the topology of the graphs of $\nu_{j}(x, t, \varepsilon)$ and $\nu_{j-1}(x, t, \varepsilon)$ allows us to move $x$ continuously to a point $x^{\prime}$ so that $\sigma_{j}$ and $\sigma_{j-1}$ do not change and either $(3.24 b)$ or $(3.24 c)$ is violated. On the other hand relation (3.21) and formula

$$
\log \left(a+\sqrt{a^{2}-\delta^{2}}\right)=-\log \left(a-\sqrt{a^{2}-\delta^{2}}\right)+2 \log \delta
$$

show that $r_{j}(x, t, \varepsilon)$ remains constant to first order during the process. Therefore,

$$
r_{j}(x, t, \varepsilon) \sim r_{j}\left(x^{\prime}, t, \varepsilon\right)
$$


and $r_{j}\left(x^{\prime}, t, \varepsilon\right)$ satisfies (3.16) because it does not satisfy all three requirements of (3.24). Q.E.D.

4. The continuum limit: definition of a density function. Heuristically our goal is to derive functions giving the density of the $\eta_{i}$ 's which have $\sigma_{i}=1$ and $\sigma_{i}=-1$ respectively. Given such functions the right-hand side of (3.9) tends to a simple Riemann integral as $\varepsilon \rightarrow 0$. We proceed rigorously following ideas of Lax and Levermore [6, I].

As a first step of the passage to the continuum limit, we express the sums in (3.9)-(3.10) as integrals over the following atomic measure in the $\eta$ variable:

$$
d \Psi_{\varepsilon}(\eta, x, t)=\varepsilon \sum_{i=1}^{N(\varepsilon)}\left[\frac{1}{2}\left(1-\sigma_{i}(x, t, \varepsilon)\right)-n_{i}(x, t, \varepsilon)\right] \delta\left(\eta-\eta_{i}\right) d \eta
$$

Here $\delta$ stands for the Dirac delta function. Equation (3.9) becomes

$$
\int^{x} u(s, t, \varepsilon) d s=4 \int_{0}^{1} \eta d \Psi_{\varepsilon}(\eta, x, t)+4 \sum_{i=1}^{N(\varepsilon)} \varepsilon \eta_{i} n_{i}(x, t, \varepsilon)+\text { const }+o(1),
$$

where "constant" means "independent of $x$ " and $o(1)$ is with respect to $\varepsilon \rightarrow 0$. Equations (3.10) become

$$
\begin{aligned}
& \int^{*} \log \left|\frac{\eta-\mu}{\eta+\mu}\right| d \Psi_{\varepsilon}(\mu, x, t)+r_{j}(x, t, \varepsilon)-r_{j}\left(x_{0}, t_{0}, \varepsilon\right)+n_{j} \gamma_{j} \\
& \quad=\eta_{j}\left(x-x_{0}\right)-4 \eta_{j}^{3}\left(t-t_{0}\right)+\int^{*} \log \left|\frac{\eta-\mu}{\eta+\mu}\right| d \Psi_{\varepsilon}\left(\mu, x_{0}, t_{0}\right)+o(1) .
\end{aligned}
$$

The asterisk in the integral sign indicates that the term corresponding to $i=j$ is excluded from the sum.

THEOREM 4.1. For $x$, $t$ fixed there is a bounded measurable function $\psi(\eta, x, t)$ such that $d \Psi_{\varepsilon} \rightarrow(1 / \pi) \psi(\eta, x, t) d \eta$ as $\varepsilon \rightarrow 0$. The limit in the sense of finite measures in $[0,1]$.

Proof. For $x, t$ fixed we show that the measures $d \Psi_{\varepsilon}$ are uniformly bounded as $\varepsilon \rightarrow 0$. Indeed it is known (and can be seen from our calculation in Appendix III) that in the sense of finite measures in [0,1]: as $\varepsilon \rightarrow 0, d \Phi_{\varepsilon}=\sum_{i=1}^{N(\varepsilon)} \delta\left(\eta-\eta_{i}\right) d \eta \rightarrow$ $(1 / \pi) \phi(\eta) d \eta$ where $\phi(\eta)$ is bounded in $[0,1]$. The uniform boundedness of the measure $d \Psi_{\varepsilon}$ follows from

$$
\left|d \Psi_{\varepsilon}\right| \leqslant \max _{i}\left\{1+\left|n_{i}(x, t, \varepsilon)\right|\right\} d \Phi_{\varepsilon} .
$$

Hence, there is a sequence $\varepsilon_{k} \rightarrow 0$ and a finite measure $d \Psi(\eta, x, t)$ such that

$$
d \Psi_{\varepsilon}(\eta, x, t) \rightarrow d \Psi(\eta, x, t) .
$$

By the Radon-Nikodým theorem, there is a measurable function $\psi(\eta, x, t)$ defined in $0 \leqslant \eta \leqslant 1$ such that

$$
d \Psi(\eta, x, t)=(1 / \pi) \psi(\eta, x, t) d \eta .
$$

The uniqueness of $\psi(\eta, x, t)$, to be shown in $\S 7$, demonstrates that the limit (4.5) exists not only in a subsequence but as $\varepsilon \rightarrow 0$. Finally (4.4) and the boundedness of $\phi(\eta)$ imply that $\psi(\eta, x, t)$ is bounded. Q.E.D. 
The boundedness of $\psi$ implies

$$
\begin{aligned}
\int^{*} \log \left|\frac{\eta_{j}-\mu}{\eta_{j}+\mu}\right| d \Psi\left(\eta_{j}, x, t\right) \\
\quad=\frac{1}{\pi} \int_{0}^{1} \log \left|\frac{\eta_{j}-\mu}{\eta_{j}+\mu}\right| \psi(\mu, x, t) d \mu+o(1) \quad(\varepsilon \rightarrow 0) .
\end{aligned}
$$

There follows

THEOREM 4.2. Equations (4.2)-(4.3) can be written in terms of $\psi$ as $\varepsilon \rightarrow 0$ :

$$
\int^{x} u(s, t, \varepsilon) d s=\frac{4}{\pi} \int_{0}^{1} \eta \psi(\eta, x, t) d \eta+4 \sum_{i=1}^{N(\varepsilon)} \varepsilon \eta_{i} n_{i}(x, t, \varepsilon)+\text { const }+o(1),
$$

$$
\begin{aligned}
& n_{j}(x, t, \varepsilon) \gamma_{j}+r_{j}(x, t, \varepsilon) \\
& \quad=\eta_{j}\left(x-x_{0}\right)-4 \eta_{j}^{3}\left(t-t_{0}\right)+\frac{1}{\pi} \int_{0}^{1} \log \left|\frac{\eta-\mu}{\eta+\mu}\right| \psi\left(\mu, x_{0}, t_{0}\right) d \mu+r_{j}\left(x_{0}, t_{0}, \varepsilon\right) \\
& \quad-\frac{1}{\pi} \int_{0}^{1} \log \left|\frac{\eta-\mu}{\eta+\mu}\right| \psi(\mu, x, t) d t+o(1) .
\end{aligned}
$$

5. The continuum limit: periodic single-well potentials. Our goal is to derive an equation for the density function $\psi(\eta, x, t)$. For the sake of simplicity, we first examine the case of initial data of the periodic single-well type as shown in Figure 5.1. We perform a W.K.B. analysis of the initial data in Appendix III with the following results:

THEOREM 5.1. Let $v(x)$ be a periodic function of period $p$ with a single well in each period as in Figure 5.1.

We assume $v(x)$ to be normalized as in (0.1c). If $\varepsilon \rightarrow 0$ and if $j=j(\varepsilon) \rightarrow \infty$ in such a way that $\eta_{j} \rightarrow \eta \in(0,1)$, then

$$
\lim \gamma_{j}=-\lim \log \delta_{j}=\int_{x_{+}}^{x_{-}+p} \sqrt{\eta^{2}-v(x)} d x=\gamma(\eta),
$$

$$
\lim \frac{1}{\varepsilon}\left(\eta_{j}-\eta_{j+1}\right)=\pi \phi(\eta)^{-1} \text { where } \phi(\eta)=\int_{x}^{x_{+}} \frac{\eta d x}{\sqrt{v(x)-\eta^{2}}}
$$

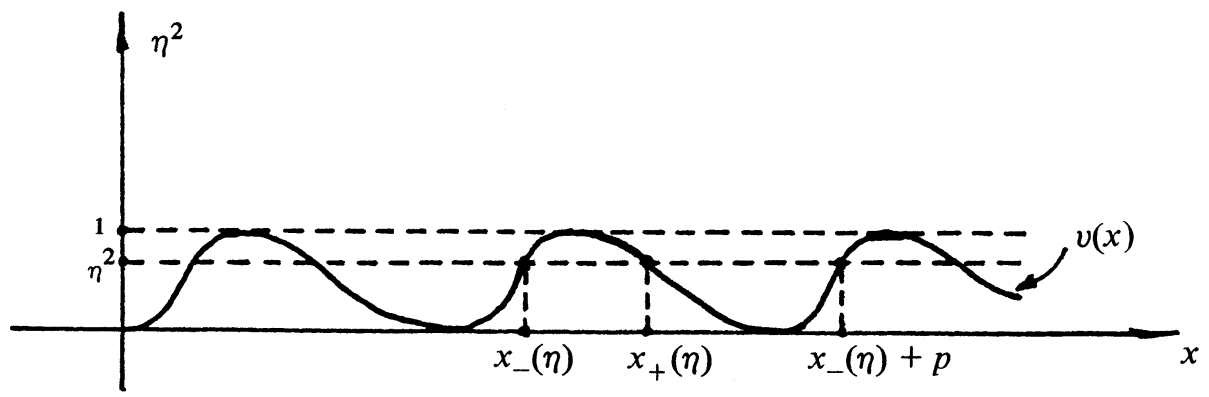

FIGURE 5.1 
Relations (5.1)-(5.2) are proven in Theorem III.4 of Appendix III. The limit of $r_{j}(x, 0, \varepsilon)$ exists and is denoted by

$$
r(\eta, x, 0)=\lim _{\varepsilon \rightarrow 0} r_{j}(x, 0, \varepsilon) .
$$

The expression for $r(\eta, x, 0)$ is given by (III.56)-(III.57) in Appendix III. The points $x_{ \pm}$are defined in Figure 5.1.

We make our expressions shorter by defining

$$
\begin{gathered}
L \psi(\eta)=\frac{1}{\pi} \int_{0}^{1} \log \left|\frac{\eta-\mu}{\eta+\mu}\right| \psi(\mu) d \mu, \\
a(\eta, x, t)=\eta\left(x-x_{0}\right)-4 \eta^{3} t-L \psi\left(\eta, x_{0}, 0\right)+r\left(\eta, x_{0}, 0\right), \\
s(\eta, x, t)=a(\eta, x, t)-L \psi(\eta, x, t) .
\end{gathered}
$$

Equations (4.9) become

$$
n_{j}(x, t, \varepsilon) \gamma\left(\eta_{j}\right)+r_{j}(x, t, \varepsilon)=s(\eta, x, t)+o(1) \quad \text { as } \varepsilon \rightarrow 0,
$$

where $j=1,2, \ldots N(\varepsilon)$.

LemMA 5.2. Let $\varepsilon \rightarrow 0$ and let $j(\varepsilon) \rightarrow \infty$ in such a way that $\eta_{j} \rightarrow \eta$. Then for all $\eta \in(0,1)$ except at most finitely many the limits

$$
n(\eta, x, t)=\lim _{\varepsilon \rightarrow 0} n_{j}(x, t, \varepsilon) \quad \text { and } \quad r(\eta, x, t)=\lim _{\varepsilon \rightarrow 0} r_{j}(x, t, \varepsilon)
$$

exist and are continuous in $\eta$.

Proof. The function $a(\eta, x, t)$ is continuous in $\eta$. The function $L \psi(\eta, x, t)$ is continuous in $\eta$ because $\psi(\eta, x, t)$ is bounded. Thus $s(\eta, x, t)$ is continuous in $\eta$. We have proven in (3.16) that $\left|r_{j}\right|<\frac{1}{2} \gamma\left(\eta_{j}\right)+o(1)$ as $\varepsilon \rightarrow 0$. Thus in the limit $\varepsilon \rightarrow 0$ relation (5.7) expresses the continuous function $s(\eta, x, t)$ as an integral number of "cycles" $\gamma(\eta)$ plus a remainder $r(\eta)$. The lemma follows immediately.

We take limits in (5.7). Following Lemma 5.2 we have

$$
a(\eta, x, t)-L \psi(\eta, x, t)=s(\eta, x, t)=n(\eta, x, t) \gamma(\eta)+r(\eta, x, t),
$$

where $n$ is an integer and $|r(\eta, x, t)| \leqslant \frac{1}{2} \gamma(\eta)$.

LeMma 5.3. Let $\varepsilon \rightarrow 0$ and $j(\varepsilon) \rightarrow \infty$ in such a way that $\eta_{j} \rightarrow \eta \in(0,1)$. For small nonzero $\varepsilon$ and for all the points $(\eta, x, t)$ at which $r(\eta, x, t)$ is continuous in $\eta$ and nonzero, the relations

$$
(5.10 \mathrm{a}-\mathrm{b}) \quad\left|\nu_{i}(x, t, \varepsilon)-\eta_{j(\varepsilon)}^{2}\right| \leqslant e^{-|r(\eta, x, t)| / \varepsilon}, \quad \sigma_{i}(x, t, \varepsilon)=\operatorname{sgn} r(\eta, x, t)
$$

hold simultaneously either when $i=j(\varepsilon)$ or when $i=j(\varepsilon)-1$.

Proof. Let $i \in\{j-1, j\}$ be defined by $\bar{D}_{i}=\max \left\{\bar{D}_{j-1}, \bar{D}_{j}\right\}$. Equation (3.13) implies that $\operatorname{sgn} r_{j}=\sigma_{i}$. Equation (5.10b) follows. We now prove (5.10a). Definition (3.7) implies

$$
\left|r_{j}\right| / \varepsilon \leqslant-\log \left\{\left|\nu_{i}-\eta_{j}^{2}\right|+\left[\left(\nu_{i}-\eta_{j}^{2}\right)^{2}-\delta_{j}^{2}\right]^{1 / 2}\right\}
$$

There follows

$$
\left|\nu_{i}-\eta_{j}^{2}\right|+\left[\left(\nu_{i}-\eta_{j}^{2}\right)^{2}-\delta_{j}^{2}\right] \leqslant e^{-r_{j} / \varepsilon}
$$


and

$$
\left|\nu_{i}-\eta_{j}^{2}\right| \leqslant e^{-\left|r_{j}\right| / \varepsilon} \rightarrow e^{-|r(\eta, x, t)| / \varepsilon} . \quad \text { Q.E.D. }
$$

TheOREM 5.4 (The BASIC INTEgRAL EQUATION). Let $n$ denote an integer and let $s(n, x, t)$ be given by (5.6). Then:

$$
\begin{gathered}
\left(n-\frac{1}{2}\right) \gamma(\eta)<s(\eta, x, t)<n \gamma(\eta) \Rightarrow \psi(\eta, x, t)=(1-n) \phi(\eta), \\
s(\eta, x, t)=n \gamma(\eta) \Rightarrow-n \phi(\eta) \leqslant \psi(\eta, x, t) \leqslant(1-n) \phi(\eta), \\
n \gamma(\eta)<s(\eta, x, t)<\left(n+\frac{1}{2}\right) \gamma(\eta) \Rightarrow \psi(\eta, x, t)=-n \phi(\eta) .
\end{gathered}
$$

Proof. We first assume that for some $(\eta, x, t)$ and for some integer $n$ :

$$
\left(n-\frac{1}{2}\right) \gamma(\eta)<s(\eta)<n \gamma(\eta) .
$$

By relation (5.9) we have

$$
n=n(\eta, x, t) \quad \text { and } \quad r(\eta, x, t)<0 .
$$

Let $j(\varepsilon) \rightarrow \infty$ in such a way that $\eta_{j} \rightarrow \eta$ as $\varepsilon \rightarrow 0$. By Lemma 5.3, for $i=j$ or $i=j-1$,

$$
\sigma_{i}(x, t, \varepsilon)=-1 \text { and }\left|\nu_{i}-\eta_{j}^{2}\right| \text { is exponentially small in } \varepsilon .
$$

As the distance of two consecutive $\eta_{j}$ 's is of order $\varepsilon$ there follows that the same $\nu_{i}$ cannot be exponentially close to two different $\eta_{j}^{2}$,s. Thus in Definition 4.1 essentially all $\sigma_{i}$ 's are equal to -1 . There follows

$$
\psi(\eta, x, t)=(1-n) \phi(\eta) .
$$

The two remaining parts follow analogously.

COROllary 5.5. Relation (5.11) can be written

$$
\psi(\eta, x, t)=-\phi(\eta) J\left\{\frac{1}{\gamma(\eta)}(a(\eta, x, t)-L \psi(\eta, x, t))\right\}
$$

where $J(w)=n$ when $w=n+\lambda, n$ integer, $0<\lambda<1$, and

$$
n-1 \leqslant J(n) \leqslant n \text { when } n \text { is an integer. }
$$

Equation (5.14) is an immediate consequence of (5.6) and (5.11).

Equation (5.14) is an integral equation for $\psi(\eta, x, t)$. It is the basic integral equation we have been searching for. For formulation of the basic integral equation as in (5.14) is preferable to (5.11) because it can be easily generalized to cover the case of general periodic potentials.

THEOREM 5.6 (THE WEAK LIMIT). The weak limit of the solution $u(x, t, \varepsilon)$ of $(0.1)$ as $\varepsilon \rightarrow 0$ in the case of initial data with a single well per period, normalized as in $(0.1 c)$ is given by

$$
\bar{u}(x, t)=\frac{\partial}{\partial x} \lim _{\varepsilon \rightarrow 0} \int^{x} u(s, t, \varepsilon) d s=\frac{4}{\pi} \frac{\partial}{\partial x} \int_{0}^{1} \eta[\psi(\eta, x, t)+n(\eta, x, t) \phi(\eta)] d \eta .
$$


Here $\psi$ is the solution to the basic integral equation (5.14) or equivalently (5.11). The integer $n(\eta, x, t)$ is determined from

$$
n(\eta, x, t) \gamma(\eta)+r(\eta, x, t)=a(\eta, x, t)-L \psi(\eta, x, t),
$$

where $a(\eta, x, t)$ is given by (5.5) and $r(\eta, x, t)$ satisfies

$$
-\frac{1}{2} \gamma(\eta) \leqslant r(\eta, x, t) \leqslant \frac{1}{2} \gamma(\eta) .
$$

The functions $\phi(\eta), \gamma(\eta)$, and $r(\eta, x, 0)$ are computed from the initial data in Appendix III.

6. General periodic initial data. We sketch the derivation of the basic integral equation in this case. We take any period $[\bar{x}, \bar{x}+p]$ of the initial data $u(x, 0)$ and we identify the endpoints to form a "circle." By the "number of wells of the initial data at $\eta^{2}$," we mean the number of disjoint intervals of the above circle on which $u(x, 0)-\eta^{2}$ is negative. We denote this number by $q(\eta)$. Asymptotically, as $\varepsilon \rightarrow 0$, the spectrum of the operator $\mathscr{L}_{\varepsilon}(0)$ near the value $\eta^{2}$ of the eigenvalue parameter is generically the union of $q(\eta)$ spectra of the form (5.1)-(5.3) having corresponding functions $\gamma_{\tau}(\eta)=\gamma(\eta), \phi_{\tau}(\eta), r_{\tau}(\eta, x, 0)$, where $\tau=1,2, \ldots q(\eta)$. These functions can be calculated by the W.K.B. method. We note that $\gamma(\eta)$ is independent of $\tau$. Corresponding to each of these, we can define the functions $a_{\tau}(\eta, x, t)$ as in (5.5) and $\psi_{\tau}(\eta, x, t)$ as in $\S 4$. The total density of spectral bands in the variable $\eta$, is given by

$$
\frac{1}{\pi \varepsilon} \phi(\eta) \quad \text { where } \phi(\eta)=\sum_{\tau=1}^{q(\eta)} \phi_{\tau}(\eta)
$$

and the total $\psi(\eta, x, t)$ defined in (4.5) is given by

$$
\psi(\eta, x, t)=\sum_{\tau=1}^{q(\eta)} \psi_{\tau}(\eta, x, t) .
$$

The limit $\varepsilon \rightarrow 0$ in equation (4.9), taken for each family of bands separately, yields

$$
L \psi(\eta, x, t)+s_{\tau}(\eta, x, t)=a_{\tau}(\eta, x, t), \quad \tau=1, \ldots, q(\eta) .
$$

An examination of the treatment in $§ 5$ shows that it can be applied to each family of bands separately. By Corollary 5.5 we obtain for $\tau=1, \ldots, q(\eta)$,

$$
\psi_{\tau}(\eta, x, t)=-\phi_{\tau}(\eta) J\left\{\frac{1}{\gamma(\eta)}\left(a_{\tau}(\eta, x, t)-L \psi(\eta, x, t)\right)\right\} .
$$

Using (6.2):

$$
\psi(\eta, x, t)=-\sum_{\tau=1}^{q(\eta)} \phi_{\tau}(\eta) J\left\{\frac{1}{\gamma(\eta)}\left(a_{\tau}(\eta, x, t)-L \psi(\eta, x, t)\right)\right\} .
$$

Equation (6.5) is an integral equation for $\psi(\eta, x, t)$ which can be solved uniquely by the method developed by Lax and Levermore in [6, II]. 
THEOREM 6.1. The weak limit of the solution $u(x, t, \varepsilon)$ of $(0.1)$ as $\varepsilon \rightarrow 0$ in the case of periodic initial data normalized as in $(0.1 \mathrm{c})$ is given by

$$
\begin{aligned}
\bar{u}(x, t) & =\frac{\partial}{\partial x} \lim _{\varepsilon \rightarrow 0} \int^{x} u(s, t, \varepsilon) d s \\
& =\frac{4}{\pi} \frac{\partial}{\partial x} \int_{0}^{1} \eta\left[\psi(\eta, x, t)+\sum_{\tau=1}^{q(\eta)} n_{\tau}(\eta, x, t) \phi_{\tau}(\eta)\right] d \eta
\end{aligned}
$$

Here $\psi$ is the solution to the basic integral equation (6.5). The integers $n_{\tau}$ are obtained from

$$
n_{\tau}(\eta, x, t) \gamma(\eta)+r_{\tau}(\eta, x, t)=a_{\tau}(\eta, x, t)-L \psi(\eta, x, t)
$$

where $a_{\tau}$ has the form of (5.5) and

$$
-\frac{1}{2} \gamma(\eta) \leqslant r_{\tau} \leqslant \frac{1}{2} \gamma(\eta), \quad \tau=1,2, \ldots q(\eta) .
$$

The functions $\phi_{\tau}(\eta), \gamma(\eta)$, and $r_{\tau}(\eta, x, 0)$ are computed from the initial data.

7. The solution of the basic integral equations. The complete solution of the basic integral equation is presented in [22]. It is obtained by its reduction to the Riemann-Hilbert problem solved by Lax and Levermore [6, II]. We describe some of the results. To be simple we work with initial data having a single well per period. For each $t \geqslant 0$ the strip of the $x-\eta^{2}$ plane $0 \leqslant \eta^{2} \leqslant 1$ is divided into two regions, one in which $\psi(\eta, x, t)$ is an integral multiple of $\phi(\eta)$ (equivalently $r(\eta, x, t) \neq 0$ ) and one in which $\psi(\eta, x, t)$ is not an integral multiple of $\phi(\eta)$ (equivalently $r(\eta, x, t)=0)$. The relevance of these relations is obvious by inspection of equation (5.11).

By the Lax-Levermore results, for each $t$, the boundary separating the two regions in the $x-\eta^{2}$ plane is a connected curve $g_{t}, p$-periodic in the $x$ variable. This curve can be specified at some $t$ as the graph of a $p$-periodic, in general multivalued, function of $x$. We denote its values by

$$
\beta_{0}^{2}(x, t)>\beta_{1}^{2}(x, t)>\cdots>\beta_{2 m}^{2}(x, t) \quad(\text { see Figure 7.1) } .
$$

They lie in the interval $[0,1]$ or in general $\left[v_{\min }, v_{\max }\right]$ if the normalization (1.c) is not adopted.

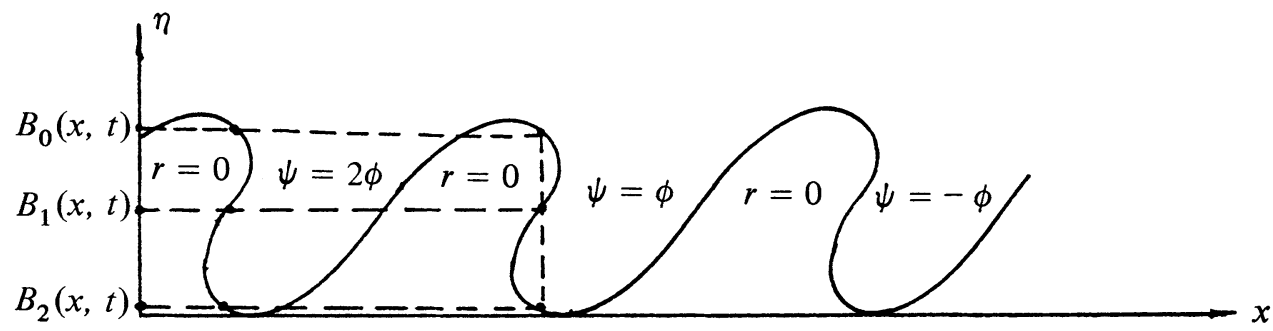


We have

$$
\begin{array}{ll}
r(\eta, x, t)=0 & \text { when } \eta \in\left[0, \beta_{2 m}\right] \cup\left[\beta_{2 m-1}, \beta_{2 m-2}\right] \cup \cdots \cup\left[\beta_{1}, \beta_{0}\right], \\
r(\eta, x, t) \neq 0 & \text { when } \eta \in\left[\beta_{2 m}, \beta_{2 m-1}\right] \cup \cdots \cup\left[\beta_{2}, \beta_{1}\right] \cup\left[\beta_{0}, \beta_{1}\right] .
\end{array}
$$

The evolution equations for the $\beta_{k}$ 's are exactly the ones derived by Lax and Levermore [equation (5.27)] in their treatment of the problem with single - well initial data:

$$
\begin{aligned}
& \left(\beta_{k}^{2 m+1}+\alpha_{2} \beta_{k}^{2 m-1}+\cdots+\alpha_{m+1} \beta_{k}\right) \frac{\partial \beta_{k}}{\partial t} \\
& +\left[12 \beta_{k}^{2 m+3}-6\left(\sum_{i=1}^{2 m+1} \beta_{i}^{2}\right) \beta_{k}^{2 m+1}+\gamma_{2} \beta_{k}^{2 m-1}+\cdots+\gamma_{m+1} \beta_{k}\right] \frac{\partial \beta_{k}}{\partial x}=0 \\
& k=0,1,2, \ldots, 2 m .
\end{aligned}
$$

The coefficients $\alpha_{i}$ 's and $\gamma_{j}$ 's are known functions of the $\beta_{i}$ 's.

At $t=0$ we have the result

$$
m(x, 0)=0 \quad \text { and } \quad \beta_{0}^{2}(x, 0)=v(x),
$$

where $v(x)=-u(x, 0)$ as in $(0.1 b)$. In the case of $m=0$ there is only $\beta_{0}$ and equations (7.3) become

$$
\partial\left(-\beta_{0}^{2}\right) / \partial t-6\left(-\beta_{0}^{2}\right) \partial\left(-\beta_{0}^{2}\right) / \partial x=0 .
$$

After equation (7.5) shocks, the curve $g_{t}$ becomes the graph of a multivalued function of $x$ and a region in the $x-t$ plane emerges having $m=1$. Equations (7.3) are in this case identical with Whitham's modulation equations [13]. For higher $m$ 's, equations (7.3) coincide with the modulation equations derived by Flaschka, Forest, and McLaughlin [4]. The connection between the $\beta_{k}$ 's and modulation theory will become apparent in the following section.

The evolution of the $\beta_{k}$ 's contains the singular phenomenon of shocking and the generation of more $\beta$ 's. Lax and Levermore circumvent this difficulty by parametrizing the curve $g_{t}$ as $x=x(s, t), \eta=\eta(s, t)$. The curve at $t=0$ is

$$
x(s, 0)=s, \quad \eta=v(s)^{1 / 2},
$$

and it evolves in time in accordance to the equations

$$
\begin{aligned}
& \eta(s, t)=\eta(s, 0)=v(s)^{1 / 2} \\
& \frac{\partial x(s, t)}{\partial t}=\left.\frac{12 \eta^{2 m+3}-6\left(\sum \beta_{k}^{2}\right) \eta^{2 m+1}+\gamma_{2} \eta^{2 m-1}+\cdots+\gamma_{m+1} \eta}{\eta^{2 m+1}+\alpha_{2} \eta^{2 m-1}+\cdots+\alpha_{m+1} \eta}\right|_{\eta=\eta(s, t)=v(s)^{1 / 2}}
\end{aligned}
$$

Once the curve $g_{t}$ is known, the function $\psi(\eta, x, t)$ is derived explicitly by the Lax-Levermore procedure. 
REMARK 7.1. At each time $t$ the quantities $m(x, t), \beta_{0}(x, t), \ldots, \beta_{2 m}(x, t)$ which appear in the right-hand side of the second equation (7.7) are functionals of the curve $g_{t}$. Their functional dependence on $g_{t}$ is given in the paragraph which contains equations (7.1). Thus, at least in principle, the system of equations (7.7) gives the evolution of the curve $g_{t}$ and consequently the evolution of the quantities $m(x, t)$ and $\left(\beta_{i}(x, t)\right)_{i=0}^{2 m}$. How to solve this system beyond breaktime explicitly is not known. Even a proof of global or even of local (beyond breaktime) existence and uniqueness of the solution of this system is still missing. These questions are important because of the physical importance of the above quantities. As we will see in $\S 8, m(x, t)$ gives the number of phases of the fast oscillation at $(x, t)$. The modulation parameters $\left(\beta_{i}\right)_{i=0}^{2 m}$ are the simple periodic/antiperiodic eigenvalues of the operator associated with the local fast oscillation. In other words the local oscillations are characterized up to phase shifts by these quantities.

8. The emergence of oscillations. We study the solution in the vicinity of a fixed point in space and time $\left(x_{0}, t_{0}\right)$. We make the change of variable

$$
x=x_{0}+\varepsilon y, \quad t=t_{0}+\varepsilon \tau,
$$

and we work in the stretched variables $y$ and $\tau$. By abuse of notation we denote $u(x, t, \varepsilon)$ and $\nu_{i}(x, t, \varepsilon)$ by $u(y, \tau, \varepsilon)$ and $\nu_{i}(y, \tau, \varepsilon)$ respectively. The $\beta_{k}$ 's are assumed to be constant in the variables $y$ and $\tau$.

THEOREM 8.1. As $\varepsilon \rightarrow 0, u(y, \tau, \varepsilon)$ has the representation

$$
u(y, \tau, \varepsilon) \sim-\beta_{0}^{2}+2 \sum_{i=1}^{m(x, t)}\left(\mathcal{N}_{i}(y, \tau, \varepsilon)-\frac{\beta_{2 i}^{2}+\beta_{2 i-1}^{2}}{2}\right),
$$

where the $\mathscr{N}_{i}^{\prime}$ s are constrained by

$$
\beta_{2 i}^{2} \leqslant \mathscr{N}_{i} \leqslant \beta_{2 i-1}^{2}
$$

and satisfy the differential equations

$$
(8.4 \mathrm{a}, \mathrm{b}) \quad \frac{\partial \mathscr{N}_{j}}{\partial y}=\frac{2 R\left(\mathscr{N}_{j}\right)^{1 / 2}}{\prod_{i \neq j}\left(\mathscr{N}_{j}-\mathscr{N}_{i}\right)}, \quad \frac{\partial \mathscr{N}_{j}}{\partial \tau}=\frac{4\left(u-2 \mathscr{N}_{j}\right) R\left(\mathscr{N}_{j}\right)^{1 / 2}}{\prod_{i \neq j}\left(\mathscr{N}_{j}-\mathscr{N}_{i}\right)}
$$

where

$$
R\left(\mathscr{N}_{j}\right)=\prod_{k=0}^{2 m}\left(\mathscr{N}_{j}-\beta_{k}^{2}\right)
$$

REMARK 8.1. Representation (8.2)-(8.5) tells us that $u(y, \tau, \varepsilon)$ is an $m$-phase quasi-periodic soliton solution of the $\mathrm{KdV}$ equation

$$
u_{\tau}-6 u u_{y}+u_{y y y}=0 .
$$

The corresponding spectral bands are the intervals $\left[\beta_{1}^{2}, \beta_{0}^{2}\right],\left[\beta_{3}^{2}, \beta_{2}^{2}\right], \ldots,\left[-\infty, \beta_{2 m}^{2}\right]$ and the corresponding Dirichlet eigenvalues are the $\mathcal{N}_{i}$ 's. The solution $u(x, t, \varepsilon)$ is thus quasiperiodic in the fast variables $x / \varepsilon, t / \varepsilon$ with the spectral parameters $\beta_{k}$ varying slowly according to (7.3). This establishes the connection between the $\beta_{k}$ 's and the modulation equation which was stated earlier. 
The proof of this theorem rests on a plausible conjecture of statistical nature which is explained in the following. To keep expressions simple we sketch the proof in the case $m=1$, in which there are only three $\beta$ 's, namely $\beta_{0}, \beta_{1}$, and $\beta_{2}$. A more detailed proof of essentially the same theorem is given in [12].

Sketch of Proof. We examine the microstructure of the $\nu_{i}$ 's

$$
\eta_{1}^{2}>\nu_{1}>\eta_{2}^{2}>\nu_{2}>\eta_{3}^{2}>\cdots>\eta_{N}^{2}>\nu_{N} \geqslant 0
$$

as defined in the second section. According to (7.2)

$$
\begin{array}{ll}
r\left(\nu_{i}, x, t\right)=0 & \text { when } \nu_{i} \in\left[0, \beta_{2}^{2}\right] \cup\left[\beta_{1}^{2}, \beta_{0}^{2}\right], \\
r\left(\nu_{i}, x, t\right) \neq 0 & \text { when } \nu_{i} \in\left(\beta_{2}^{2}, \beta_{1}^{2}\right) \cup\left(\beta_{0}^{2}, 1\right) .
\end{array}
$$

Study of region with $r \neq 0$. Lemma 5.3 implies

$$
\nu_{i} \in\left(\beta_{0}^{2}, 1\right) \Rightarrow \nu_{i}=\eta_{i}^{2}+\text { error exponentially small in } \varepsilon .
$$

The contribution of the $\nu_{i}$ 's in $\left(\beta_{0}^{2}, 1\right)$ to the sum in (1.2) is

$$
2 \sum_{\left\{i: \nu_{i} \in\left(\beta_{0}^{2}, 1\right)\right\}}\left\{\nu_{i}-\frac{\xi_{2 i-1}+\xi_{2 i}}{2}\right\} \sim 1-\beta_{0}^{2} \text { as } \varepsilon \rightarrow 0 .
$$

In the region $\left(\beta_{2}^{2}, \beta_{1}^{2}\right)$ where again $r \neq 0$, not all $\nu_{i}$ 's can be determined through Lemma 5.3. The theorem dictates the configuration of Figure 8.1 in which one $\nu_{i}$ denoted by $\mathscr{N}$ does not need to be close to some $\eta_{j}^{2}$.

In this figure an interval denotes a band, a dot denotes a $\nu_{i}$, and a dot touching a band indicates that the distance between the corresponding band and $\nu_{i}$ decays exponentially as $\varepsilon \rightarrow 0$. As $y$ and $\tau$ vary, $\mathscr{N}$ oscillates in the interval $\left[\beta_{2}^{2}, \beta_{1}^{2}\right]$ effectively passing from one gap to its neighboring one as shown in Figure 8.1.

The contribution to (1.2) from the region $\left(\beta_{2}^{2}, \beta_{1}^{2}\right)$ as $\varepsilon \rightarrow 0$ is

$$
2 \sum_{\left\{i: \nu_{i} \in\left(\beta_{2}^{2}, \beta_{1}^{2}\right)\right\}}\left(\nu_{i}-\frac{\xi_{2 i-1}+\xi_{2 i}}{2}\right) \sim\left(-\beta_{1}^{2}-\mathscr{N}\right)+\left(\mathscr{N}-\beta_{2}^{2}\right)=2 \mathscr{N}-\beta_{1}^{2}-\beta_{2}^{2} \text {. }
$$

Study of region $r=0$. We make the following assumption which we can prove at $t=0$.

Assumption of molecular chaos. If $r(\eta, x, t)=0$, there is $\varepsilon \ll l(\varepsilon) \ll 1$ as $\varepsilon \rightarrow 0$ such that

$$
\sum_{\left\{i: \eta^{2} \leqslant \nu_{i} \leqslant \eta^{2}+l(\varepsilon)\right\}}\left(\nu_{i}-\frac{\xi_{2 i}+\xi_{2 i-1}}{2}\right) \ll l(\varepsilon) \text { as } \varepsilon \rightarrow 0
$$

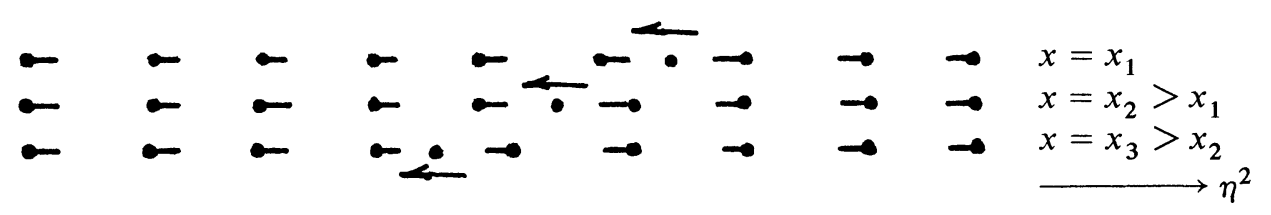

FIGURE 8.1 
In other words, in the regions having $r=0$ the local average of the deviation of the $\nu_{i}$ from the center of its interval is of order lower than $\varepsilon$. The assumption gives immediately

$$
\sum_{\left\{i: r\left(\nu_{i}\right)=0\right\}}\left(\nu_{i}-\frac{\xi_{2 i}+\xi_{2 i-1}}{2}\right) \ll 1 \quad \text { as } \varepsilon \rightarrow 0 .
$$

Using (8.9), (8.10), and (8.12) in (1.2) where $\xi_{0}=1$ we obtain the result (8.2).

The proof of (8.4) goes along similar lines. For example, to prove (8.4a) we start from representation (1.3)-(1.4) of the solution $u(x, t, \varepsilon)$. Neglecting all the $\nu_{i}$ 's for $i>N($ see $(2.5))$ we obtain

$$
\frac{\partial \nu_{i}}{\partial y}=\frac{2\left\{\prod_{k=0}^{2 N}\left|\xi_{k}-\nu_{i}\right|\right\}^{1 / 2}}{\prod_{k=1 ; k \neq i}^{N}\left(\nu_{i}-\nu_{k}\right)}, \quad i=1,2, \ldots, N
$$

We apply this to $\nu_{i}=\mathscr{N}$

$$
\frac{\partial \mathscr{N}}{\partial y}=\frac{2\left\{\prod_{k=0}^{2 N}\left|\xi_{k}-\mathscr{N}\right|\right\}^{1 / 2}}{\prod_{k=1 ; k \neq i}^{N}\left(\mathscr{N}-\nu_{k}\right)}
$$

We perform the following cancellations:

(a) $\left\{\nu_{k}: r\left(\nu_{k}, x, t\right) \neq 0\right\}$. By Lemma 5.3 we have

$$
\tilde{\boldsymbol{\nu}}_{k}=\text { either } \nu_{k} \text { or } \nu_{k-1}=\eta_{k}^{2}+\text { error exponentially small as } \varepsilon \rightarrow 0 \text {. }
$$

Thus

$$
\frac{\left\{\left|\xi_{2 k-1}-\mathscr{N}\right|\left|\xi_{2 k-2}-\mathscr{N}\right|\right\}^{1 / 2}}{\left|\mathcal{N}-\tilde{\nu}_{k}\right|}=1+O\left(\varepsilon^{-M}\right) \text { for any integer } M
$$

(b) $\left\{\nu_{i}: r\left(\nu_{k}, x, t\right)=0\right\}$. In this region as $\varepsilon \rightarrow 0$

$$
\frac{\left\{\left|\xi_{2 k-1}-\mathscr{N}\right|\left|\xi_{2 k}-\mathscr{N}\right|\right\}^{1 / 2}}{\left|\mathscr{N}-\nu_{k}\right|} \sim 1-\frac{\nu_{k}-\frac{1}{2}\left(\xi_{2 k-1}+\xi_{2 k}\right)}{\mathscr{N}-\nu_{k}}+O\left(\varepsilon^{2}\right)
$$

By the molecular chaos assumption the product of these factors tends to 1 as $\varepsilon \rightarrow 0$. The factors which remain after cancellations (a) and (b) have been performed are exactly the factors in the right-hand side of (8.4a). Q.E.D.

There is still one piece of information which eludes us. We do not have an initial condition for equations (8.4); therefore we know the solution only up to phase shifts. To capture the phase shifts one must solve equations (1.12) not only to leading order but to order $\varepsilon$ as $\varepsilon \rightarrow 0$. This follows from the fact that a change in $x$ of the order of the wavelength of the oscillations, i.e., a change of order $\varepsilon$ in $x$, produces a change of order $\varepsilon$ in the right-hand side of (1.12). The same order of error is introduced by our molecular chaos assumption and by various other manipulations we have performed. 


\section{Appendix I: Proof of Theorem 1.1.}

LEMMA. For $g$ finite or infinite,

$$
\begin{aligned}
A= & \sum_{i=1}^{g} \frac{2 \pi^{2} i^{2}}{p^{2}} \frac{P\left(\nu_{i}\right)}{W_{i}\left(\nu_{1}, \nu_{2}, \ldots\right)}=-\xi_{0}+2 \sum_{i=0}^{g}\left(\nu_{i}-\frac{\xi_{2 i-1}+\xi_{2 i}}{2}\right), \\
B= & \sum_{i=1}^{g} \frac{\pi^{2} i^{2}}{p^{2}} \frac{P_{j}\left(\nu_{i}\right)}{W_{i}\left(\nu_{1}, \nu_{2}, \ldots\right)}=1 \\
C= & \sum_{i=1}^{g} \frac{2 \pi^{2} i^{2}}{p^{2}} \frac{P_{j}\left(\nu_{i}\right)}{W_{i}\left(\nu_{1}, \nu_{2}, \ldots\right)} \\
& \times\left[\xi_{0}-\xi_{2 i}-\xi_{2 i-1}+2 \sum_{\substack{k=1 \\
k \neq i}}^{g}\left(\nu_{k}-\frac{\xi_{2 k-1}+\xi_{2 k}}{2}\right)\right]=-4 \rho_{j} .
\end{aligned}
$$

Proof. We first prove the lemma for finite $g$. To avoid lengthy expressions we set

$$
D_{i}=\prod_{\substack{k=1 \\ k \neq i}}^{g}\left(\nu_{i}-\nu_{k}\right)
$$

We will make heavy use of the following identity which is a consequence of the Lagrange interpolation formula:

$$
\sum_{i=1}^{g} \frac{\nu_{i}^{\tau}}{D_{i}}= \begin{cases}\sum_{i=1}^{g} \nu_{i} & \text { when } \tau=g \\ 1 & \text { when } \tau=g-1 \\ 0 & \text { when } \tau<g-1\end{cases}
$$

where $g, \tau$ are natural numbers.

(a)

$$
A=2 \sum^{g} \frac{\prod_{k=1}^{g}\left(\nu_{i}-\rho_{k}\right)}{D_{i}}=2 \sum_{i=1}^{g} \frac{\nu_{i}^{g}-\left(\sum_{k=1}^{g} \rho_{k}\right) \nu_{i}^{g-1}+\cdots}{D_{i}}=2 \sum_{i=1}^{g} \nu_{i}-2 \sum_{k=1}^{g} \rho_{k} .
$$

We take the value of $\sum_{k=1}^{g} \rho_{k}$ from (1.10a) to obtain (I.1).

(b)

$$
B=\sum_{i=1}^{g} \frac{\prod_{k=1 ; k \neq j}^{g}\left(\nu_{i}-\rho_{k}\right)}{D_{i}}=\sum_{i=1}^{g} \frac{\nu_{i}^{g-1}+\cdots}{D_{i}}=1
$$

(c)

$$
\begin{aligned}
C & =2 \sum_{i=1}^{g} \frac{\left(-\sum_{k=0}^{g} \xi_{k}+2 \sum_{k=1}^{g} \nu_{k}-2 \nu_{i}\right)}{D_{i}} \prod_{\substack{k=1 \\
k \neq j}}^{g}\left(\nu_{j}-\rho_{k}\right) \\
& =2 \sum_{i=1}^{g} \frac{\left[\left(-\sum_{k=0}^{g} \xi_{k}+2 \sum_{k=1}^{g} \nu_{k}\right)-2 \nu_{i}\right]\left[\nu_{i}^{g-1}+\left(\rho_{j}-\sum_{k=1}^{g} \rho_{k}\right) \nu_{i}^{g-2}+\cdots\right]}{D_{i}} \\
& =-4 \sum_{i=1}^{g} \frac{\nu_{i}^{g}}{D_{i}}+2\left[-\sum_{k=0}^{g} \xi_{k}+2 \sum_{k=1}^{g} \nu_{k}-2 \rho_{j}+2 \sum_{k=1}^{g} \rho_{k}\right] \sum_{i=1}^{g} \frac{\nu_{i}^{g-1}}{D_{i}}+\cdots
\end{aligned}
$$


By (I.5)

$$
C=-4 \sum_{i=1}^{g} \nu_{i}-2 \sum_{k=0}^{g} \xi_{k}+4 \sum_{k=1}^{g} \nu_{k}-4 \rho_{j}+4 \sum_{k=1}^{g} \rho_{k} .
$$

When the value of $\sum_{k=1}^{g} \rho_{k}$ is substituted from (1.10a) we obtain $C=-4 \rho_{j}$.

Thus (I.1)-(I.3) have been shown for $g$ finite. To prove the case $g=\infty$ we write $A, B$, and $C$ as $A=A_{N}+r_{N}^{A}, B=B_{N}+r_{N}^{B}$, and $C=C_{N}+r_{N}^{C}$ where $A_{N}, B_{N}, C_{N}$ are obtained by truncating the products in the definition of $P$ and $W$ and the sums in the definition of $A, B, C$ at $i=N . A_{N}, B_{N}, C_{N}$ satisfy (I.1), (I.2), (I.3) respectively, and there only remains to be shown that $r_{N}^{A}, r_{N}^{B}, r_{N}^{C} \rightarrow 0$ as $N \rightarrow \infty$. We prove this for $r_{N}^{A}$ only, the proofs for $r_{N}^{B}, r_{N}^{C}$ being similar.

The $i$ th term in the series for $A$ can be written

$$
\begin{aligned}
\frac{\pi^{2} i^{2}}{p^{2}} \frac{P\left(\nu_{i}\right)}{W_{i}\left(\nu_{1}, \ldots\right)} & =\left(\nu_{i}-\rho_{i}\right) \prod_{k=1}^{\infty}, \frac{\nu_{i}-\rho_{k}}{\nu_{i}-\nu_{k}} \\
& =\left(\nu_{i}-\rho_{i}\right) \prod_{k=1}^{N}, \frac{\nu_{i}-\rho_{k}}{\nu_{i}-\nu_{k}}+E_{i N},
\end{aligned}
$$

where the prime ' indicates that the factor corresponding to $k=i$ is omitted from the product. We have

$$
\begin{aligned}
A & =\sum_{i=1}^{\infty}\left\{\left(\nu_{i}-\rho_{i}\right) \prod_{k=1}^{N}, \frac{\nu_{i}-\rho_{k}}{\nu_{i}-\nu_{k}}+E_{i N}\right\} \\
& =\underbrace{\sum_{i=1}^{N}\left(\nu_{i}-\rho_{i}\right) \prod_{k=1}^{N}, \frac{\nu_{i}-\rho_{k}}{\nu_{i}-\nu_{k}}}_{A_{N}}+\underbrace{\sum_{i=N+1}^{\infty}\left(\nu_{i}-\rho_{i}\right) \prod_{k=1}^{N} \frac{\nu_{i}-\rho_{k}}{\nu_{i}-\nu_{k}}+\sum_{i=1}^{\infty} E_{i N} .}_{r_{N}^{A}}
\end{aligned}
$$

The first term of the remainder $r_{N}^{A}$ converges to zero as $N \rightarrow \infty$ because the product tends to 1 and the difference $\rho_{i}-\nu_{i}$ decays fast when $i>N \rightarrow \infty$ (see (1.7)-(1.8)). To study the second term of $r_{N}^{A}$ we estimate the remainder $E_{i N}$ :

$$
E_{i N}=\left(\nu_{i}-\rho_{i}\right)\left\{\prod_{k=1}^{\infty} \prime^{\prime}(\cdot)-\prod_{k=1}^{N}{ }^{\prime}(\cdot)\right\}=\left(\nu_{i}-\rho_{i}\right) \prod_{k=1}^{N}{ }^{\prime}(\cdot)\left\{\prod_{k=N+1}^{\infty}{ }^{\prime}(\cdot)-1\right\} .
$$

The products in (I.8) are bounded uniformly in $i, N$ so

$$
\left|E_{i N}\right| \leqslant \text { Const }\left|\nu_{i}-\rho_{i}\right| \text {. }
$$

On the other hand when $i<N$

$$
\begin{aligned}
\left|E_{i N}\right| & \leqslant \text { Const }\left|1-\prod_{k=N+1}^{\infty}\left(\frac{\nu_{i}-\rho_{k}}{\nu_{i}-\nu_{k}}\right)\right| \\
& \leqslant \text { Const } \sum_{k=N+1}^{\infty}\left|\frac{\nu_{k}-\rho_{k}}{\nu_{i}-\nu_{k}}\right| \leqslant \frac{\text { Const }}{\left|\nu_{i}-\nu_{N}\right|} \sum_{k=N+1}^{\infty}\left|\nu_{k}-\rho_{k}\right| .
\end{aligned}
$$

Estimates (I.9)-(I.10) yield

$$
\sum_{i=0}^{\infty}\left|E_{i N}\right| \leqslant \text { Const } \sum_{i=1}^{N} \frac{1}{\left|\nu_{i}-\nu_{N}\right|} \sum_{k=N+1}^{\infty}\left|\nu_{k}-\rho_{k}\right|+\text { Const } \sum_{i=N+1}^{\infty}\left|\nu_{i}-\rho_{i}\right| .
$$


As $N \rightarrow \infty$, the right-hand side of (I.11) approaches zero because of the fast decay of $\left|\nu_{k}-\rho_{k}\right|$ (see (1.7)-(1.8)). We have shown that $r_{N}^{A} \rightarrow 0$ as $N \rightarrow \infty$. The proof of the lemma is complete.

Proof of TheOrem. The first equality (1.11) is a repetition of (1.2). Using (I.1) and (1.3) we obtain

$$
u(x, t)=\sum_{i=1}^{g} \frac{2 \pi^{2} i^{2}}{p^{2}} \frac{P\left(\nu_{i}\right)}{W_{i}\left(\nu_{1}, \ldots\right)}=\sum_{i=1}^{g} \frac{P\left(\nu_{i}\right)}{R\left(\nu_{i}\right)^{1 / 2}} \frac{\partial \nu_{i}}{\partial x},
$$

The second equality (1.11) follows immediately. To prove (1.12) we obtain by the use of (1.3)-(1.4) and (I.2)-(I.3) and by an easy calculation

$$
\begin{gathered}
\sum_{i=1}^{g} \frac{P_{j}\left(\nu_{i}\right)}{R\left(\nu_{i}\right)^{1 / 2}} \frac{\partial \nu_{i}}{\partial x}=2, \\
\sum_{i=1}^{g} \frac{P_{j}\left(\nu_{i}\right)}{R\left(\nu_{i}\right)^{1 / 2}} \frac{\partial \nu_{i}}{\partial t}=-8 \rho_{j},
\end{gathered}
$$

which can be rewritten as

$$
\begin{gathered}
\frac{\partial}{\partial x} \sum_{i=1}^{g} \int^{\nu_{i}(x, t)} \frac{P_{j}(\mu)}{R(\mu)^{1 / 2}} d \mu=2, \\
\frac{\partial}{\partial t} \sum_{i=1}^{g} \int^{\nu_{i}(x, t)} \frac{P_{j}(\mu)}{R(\mu)^{1 / 2}} d \mu=-8 \rho_{j} .
\end{gathered}
$$

(1.12) follows immediately. This concludes the proof of the theorem.

\section{Appendix II.}

1. Calculation of $A(\nu)$. In relation (3.1a) $P(\nu)$ is given by (1.9) and $R(\nu)$ is given by (1.5). We set

$$
2 \Gamma_{i}= \begin{cases}\text { width of } i \text { th band } & \text { when } i \leqslant N \\ \text { width of } i \text { th gap } & \text { when } i>N .\end{cases}
$$

Using (2.4) and (2.5) we obtain easily

$$
|A(\nu)|=\int^{\nu}|\nu|^{-1 / 2} \prod_{i=1}^{g} \frac{\left|\nu-\rho_{i}\right|}{\left|\left(\nu-\rho_{i}\right)^{2}-\Gamma_{i}^{2}\right|^{1 / 2}} d \nu .
$$

The $\Gamma_{i}$ 's are exponentially small in $\varepsilon$, when the corresponding $\rho_{i}$ 's are bounded away from zero, and they are of order $\varepsilon$ when the corresponding $\rho_{i}$ 's are in a neighborhood of zero which shrinks as $\varepsilon \rightarrow 0$. It is easily seen that the infinite product in the integrand of (II.1) equals $1+o(1)$ as $\varepsilon \rightarrow 0$ unless:

(a) $\nu$ is exponentially close to some $\rho_{i}$ and $\Gamma_{i}$ cannot be neglected, or

(b) $\nu$ is in a neighborhood of zero.

We assume the product to be equal to 1 to first order, because the error in $A(\nu)$ produced by (a) is eliminated to all $\varepsilon$ orders by the integration in (II.1), while the error due to (b) produces a total of error of order $o(1)$ in (3.3) as $\varepsilon \rightarrow 0$. The former claim is trivial to verify, while the latter one involves showing that the region around 
$\nu=0$ in which the $\Gamma_{i}$ 's are of order $\varepsilon$ shrinks fast enough as $\varepsilon \rightarrow 0$. This can be verified using the asymptotic results on bands and gaps obtained by Weinstein and Keller [10].

Setting the product in (II.1) equal to 1, using the integration constants as in (3.2c), and choosing signs by (3.1c) we see that, for $\nu$ in the gap region, $A(\nu)$ can be replaced by

$$
\bar{A}(\nu)= \begin{cases}-2 \nu^{1 / 2} & \text { when } \nu>0 \\ 0 & \text { when } \nu<0\end{cases}
$$

2. Calculation of $D_{j}(\nu)$. A consequence of (II.3) is that the terms corresponding to negative $\nu_{i}$ 's in (3.3) can be neglected. Having eliminated some unknowns in this way, we retain in system (3.5) only these equations corresponding to $j \leqslant N$. Thus we need to calculate $D_{j}(\nu)$ only when $j \leqslant N$. Again we calculate only for $\nu$ in the gaps. As in the calculation of $A(\nu)$ we obtain

$$
\left|D_{j}(\nu)\right|=\int^{\nu} \frac{|\nu|^{-1 / 2}}{\left|\nu-\rho_{j}\right|} \prod_{i=1}^{\infty} \frac{\left|\nu-\rho_{i}\right|}{\left|\left(\nu-\rho_{i}\right)^{2}-\Gamma_{i}^{2}\right|^{1 / 2}} d \nu .
$$

Case 1. $\left|\nu-\rho_{j}\right|$ not too small, say $\left|\nu-\rho_{j}\right|>\varepsilon^{2}$. As in the calculation of $A(\nu)$ we set the product in the integrand of (II.4) equal to 1 . We integrate using the calculus formula

$$
\int \frac{\nu^{-1 / 2}}{\nu-\rho_{j}} d \nu=\frac{1}{\rho_{j}^{1 / 2}} \log \left|\frac{\nu^{1 / 2}-\rho_{j}^{1 / 2}}{\nu^{1 / 2}+\rho_{j}^{1 / 2}}\right|+\text { const. }
$$

We use (3.1c) for the determination of the sign and (3.2d) to determine the constant. We obtain the approximate $D_{j}$ :

$$
\begin{gathered}
\bar{D}_{j}(\nu)=-\frac{1}{\rho_{j}^{1 / 2}} \log \left|\frac{\nu^{1 / 2}-\rho_{j}^{1 / 2}}{\nu^{1 / 2}+\rho_{j}^{1 / 2}}\right| \text { when } \nu>0(\varepsilon \rightarrow 0), \\
\bar{D}_{j}(\nu)=0 \quad \text { when } \nu<0 .
\end{gathered}
$$

Remark. For $\nu<0$ (and $\nu$ in a gap) $D_{j}(\nu)$ decays exponentially as $\varepsilon \rightarrow 0$ because by the choice of integration constant it is zero in the midpoint of the gap and the gap in this region is exponentially small. On the other hand the $m_{i}$ 's which multiply $D_{j}$ in (3.5) are $O(1 / \varepsilon)$ by (3.14). Thus the approximation $\bar{D}_{j}(\nu)=0$ when $\nu<0$ is justified.

Case 2. $\left|\rho_{j}-\nu\right|<\varepsilon^{2}$. By setting the product in (II.4), with the exception of the factor corresponding to $i=j$, equal to 1 we obtain

$$
\left|\bar{D}_{j}(\nu)\right|=\int^{\nu} \frac{\left|\nu^{-1 / 2}\right|}{\left|\left(\nu-\rho_{j}\right)^{2}-\Gamma_{j}^{2}\right|^{1 / 2}} d \nu .
$$

By integration and use of (3.1c) we obtain

$$
\bar{D}_{j}(\nu)=-\frac{1}{\rho_{j}^{1 / 2}} \log \left(\left|\nu-\rho_{j}\right|+\left[\left(\nu-\rho_{j}\right)^{2}-\Gamma_{j}^{2}\right]^{1 / 2}\right)+\text { const. }
$$


$\bar{D}_{j}(\nu)$ satisfies all conditions (3.2b). Moreover, (II.5a) and (II.5b) match if a constant $\sim-\log \left(1 / 8 \rho_{j}^{3 / 2}\right)$ is chosen (II.5b). This constant would be negligible in (3.5) and is omitted.

The calculation of the approximate $A(\nu)$ and $D(\nu)$ is now complete.

\section{Appendix III.}

The spectral data. We assume that the initial function $u(x, 0, \varepsilon)=-v(x)$ of $(0.1)$ satisfies

$$
v(x)=v(x+p)
$$

and is normalized so that

$$
\max v(x)=1, \quad \min v(x)=0 .
$$

We study the eigenvalue problem

$$
\mathscr{L}_{\varepsilon}(0) f(s)=\varepsilon^{2} f^{\prime \prime}(s)-u(s, 0, \varepsilon) f(s)=\eta^{2} f(s),
$$

or equivalently

$$
\varepsilon^{2} f^{\prime \prime}(s)+v(s) f(s)=\eta^{2} f(s)
$$

with two types of boundary conditions:

(a) Periodic/Antiperiodic. $f(x)=\alpha f(x+p)$ and

$$
f^{\prime}(x)=\alpha f^{\prime}(x+p) \quad \text { where } \alpha= \pm 1 .
$$

(b) Dirichlet.

$$
f(x)=f(x+p)=0 .
$$

It is convenient to make the change of variable: $k^{2}=1-\eta^{2}$ and to work with the potential $1+v(s)$.

Equation (III.1) can be written

$$
-\varepsilon^{2} f^{\prime \prime}(s)+[1+v(s)] f(s)=k^{2} f(s) .
$$

The eigenvalue problems are defined for $x \leqslant s \leqslant x+p$. We extend the validity of (III.4) to the whole line $-\infty<s<\infty$ by extending the potential $1+v(s)$ as follows:

$$
q(s)= \begin{cases}1+v(s) & \text { when } x \leqslant s \leqslant x+p, \\ 0 & \text { otherwise }\end{cases}
$$

The boundary conditions (II.2)-(III.5) can be expressed in terms of the entries of the scattering matrix of the extended potential. These are the transmission coefficient $T(k, x, \varepsilon)$ and the left reflection coefficient defined by the asymptotic relation

$$
f(s, k, x, \varepsilon)= \begin{cases}\frac{e^{i k s / \varepsilon}}{T(k, x, \varepsilon)}+\frac{R(k, x, \varepsilon)}{T(k, x, \varepsilon)} e^{-i k s / \varepsilon} & \text { when } s \leqslant x \\ e^{i k s / \varepsilon} & \text { when } s \geqslant x+p\end{cases}
$$


where $f$ is a solution of

$$
-\varepsilon^{2} f^{\prime \prime}(s)+q(s) f(s)=k^{2} f(s), \quad-\infty<s<\infty .
$$

We let

$$
\begin{aligned}
& T(k, x, \varepsilon)=\exp [-\alpha(k, x, \varepsilon)+i \tau(k, x, \varepsilon)], \\
& R(k, x, \varepsilon)=\exp [-r(k, x, \varepsilon)+i \rho(k, x, \varepsilon)],
\end{aligned}
$$

where $\alpha, \tau, r$ and $\rho$ are real.

We will subsequently use the well-known fact

$$
e^{-2 \alpha}+e^{-2 r}=1 \text {. }
$$

THEOREM III.1 (J. B. KELLER). The periodic/antiperiodic eigenvalues of $\mathscr{L}_{\varepsilon}(0)$ are given by $\xi_{i}=1-k_{i}^{2}$ where $k_{i}$ are the solutions of the equation

$$
\cos (k p / \varepsilon+\tau)= \pm e^{-\alpha} .
$$

This is an immediate consequence of a theorem by Keller [5]. It is known that the $\xi_{i}$ 's are independent of $x$ and that $\left[\xi_{2 i-1}, \xi_{2 i-2}\right]$ is the $i$ th spectral band of the selfadjoint operator $\mathscr{L}_{\varepsilon}(0)$ in the line $-\infty<s<\infty$.

THEOREM III.2. The Dirichlet eigenvalues of $\mathscr{L}_{\varepsilon}(0)$ are given by: $\nu_{i}(x)=1-k_{i}^{2}$ where $k_{i}$ are the solutions of the equation

$$
\sin (k p / \varepsilon+\tau)+e^{-r} \sin (k p / \varepsilon+\tau+2 k x / \varepsilon-\rho)=0,
$$

where $\rho$ and $\tau$ are defined by (III.8)-(III.9).

Proof. Let $g(s, k, x, \varepsilon)=f(s, k, x, \varepsilon) e^{-i k(x+p) / \varepsilon}$ where $f$ is given by (III.6).

$$
g(s, k, x, \varepsilon)= \begin{cases}\frac{e^{i k(s-x-p) / \varepsilon}}{T}+\frac{R}{T} e^{-i k(s+x+p) / \varepsilon} & \text { when } s \leqslant x, \\ e^{i k(s-x-p) / \varepsilon} & \text { when } s \geqslant x+p .\end{cases}
$$

The real and imaginary parts of $g$ also satisfy (III.7) when $k^{2}$ is real. Furthermore,

$$
\begin{gathered}
\left.\operatorname{Im} g\right|_{s=x+p}=0, \\
\left.\frac{1}{2} \operatorname{Im} g\right|_{s=x}=e^{\alpha} \sin (-k p / \varepsilon-\tau)+e^{\alpha-r} \sin (-k p / \varepsilon-2 k x / \varepsilon+\rho-\tau) .
\end{gathered}
$$

We obtain the Dirichlet eigenvalues by setting $\left.\operatorname{Im} g\right|_{s=x}=0$. Hence,

$$
\sin (k p / \varepsilon+\tau)+e^{-r} \sin (k p / \varepsilon+\tau+2 k x / \varepsilon-\rho)=0 . \quad \text { Q.E.D. }
$$

We now calculate explicitly the transmission and reflection coefficients $T(k, x, \varepsilon)$, $R(k, x, \varepsilon)$. We restrict ourselves to the case in which $v(x)$ is a smooth $p$-periodic function which has exactly one maximum and exactly one minimum in each period $[x, x+p)$. The calculation is performed in the following lemma. 
LeMmA III.3. Let $0<k^{2}<1$.

Case 1. $q(x)<k^{2}$. (Equivalently $\left.v(x)>\eta^{2}\right)$. As $\varepsilon \rightarrow 0$ :

$$
\begin{gathered}
\rho(k, x, \varepsilon) \sim 2 J / \varepsilon-\pi / 2+O\left(\varepsilon^{2}\right), \\
\tau(k, x, \varepsilon) \sim(I+J) / \varepsilon+O\left(\varepsilon^{2}\right), \\
\alpha(k, x, \varepsilon) \sim \Gamma / \varepsilon+O\left(\varepsilon^{2}\right),
\end{gathered}
$$

where

(III.14a)

$$
J(k, x)=x k+\int_{x}^{x_{1}}\left(k^{2}-q(y)\right)^{1 / 2} d y,
$$

$$
I(k, x)=-(x+p) k+\int_{x_{2}}^{x+p}\left(k^{2}-q(y)\right)^{1 / 2} d y,
$$

$$
\Gamma(k)=\int_{x_{1}}^{x_{2}}\left(q(y)-k^{2}\right)^{1 / 2} d y
$$

and $x_{1}(k)<x_{2}(k)$ denote the two solutions of the equation

$$
q(y)=k^{2}, \quad x \leqslant y<x+p .
$$

Case 2. $k^{2}<q(x)$. (Equivalently $v(x)<\eta^{2}$.) As $\varepsilon \rightarrow 0$

$$
\tau(k, x, \varepsilon)=-\frac{k p}{\varepsilon}+\frac{\Theta}{\varepsilon}-\arg \left\{1+\left[1+\frac{e^{-L_{1} / \varepsilon}+e^{-L_{2} / \varepsilon}}{2}\right]\right\} e^{2 i \Theta / \varepsilon}+O\left(\varepsilon^{2}\right),
$$

(III.16b) $\tau(k, x, \varepsilon)-\rho(k, x, \varepsilon)=-\frac{2 x k+k p}{\varepsilon}+\frac{\Theta(k)}{\varepsilon}$

$$
-\arg \left\{1+\left[1+\frac{e^{-L_{1} / \varepsilon}-e^{-L_{2} / \varepsilon}}{2}\right]\right\} e^{2 i \Theta / \varepsilon}+O\left(\varepsilon^{2}\right),
$$

(III.16c) $\alpha(k, x, \varepsilon) \sim \frac{L_{1}+L_{2}}{\varepsilon}+\log \left|1+\left[1+\frac{e^{-L_{1} / \varepsilon}+e^{-L_{2} / \varepsilon}}{2}\right] e^{2 i \Theta / \varepsilon}\right|$,

where

(III.17a)

(III.17b)

(III.17c)

$$
\begin{aligned}
L_{1}(k, x) & =\int_{x}^{x_{2}}\left(q(y)-k^{2}\right)^{1 / 2} d y, \\
L_{2}(k, x) & =\int_{x_{1}}^{x+p}\left(q(y)-k^{2}\right)^{1 / 2} d y, \\
\Theta(k) & =\int_{x_{2}}^{x_{1}}\left(k^{2}-q(y)\right)^{1 / 2} d y,
\end{aligned}
$$

and $x_{2}(k)<x_{1}(k)$ denote the two solutions of

$$
q(y)=k^{2}, \quad x \leqslant y<x+p .
$$


Proof. The formulas for Case 1 are well known, see e.g. [1]. We derive the formulas in the second case by letting

$$
q(y)=q_{1}(y)+q_{2}(y)
$$

where

$$
\begin{gathered}
q_{1}(y)= \begin{cases}q(y) & \text { when } x \leqslant y \leqslant c, \\
0 & \text { otherwise, }\end{cases} \\
q_{2}(y)= \begin{cases}q(y) & \text { when } c \leqslant y \leqslant x+p \\
0 & \text { otherwise. }\end{cases}
\end{gathered}
$$

$c$ is the unique point in $[x, x+p]$ for which $q(c)=0$. We call $x_{2}$ and $x_{1}$ the unique solutions of equations $q_{1}(x)=k^{2}, q_{2}(x)=k^{2}$ respectively (see Figure 1). Let $T_{i}=e^{-\alpha_{i}+i \tau_{i}}$ and $R_{i}=e^{-r_{i}+i \rho_{i}}$ be the transmission and reflection coefficients of the potentials $q_{i}(s)$ where $i=1,2$. We calculate the transmission coefficient $T(k, x, \varepsilon)$ and the reflection coefficient $R(k, x, \varepsilon)$ of $q(s)$ by considering the eigenfunction

(III.20)

$$
\psi=\left\{\begin{array}{l}
e^{i k s / \varepsilon} \quad \text { when } s \geqslant x+p, \\
\frac{e^{i k s / \varepsilon}}{T_{2}}+\frac{R_{2}}{T_{2}} e^{-i k s / \varepsilon} \quad \text { when } s=c \\
\frac{1}{T_{2}}\left(\frac{e^{i k s / \varepsilon}}{T_{1}}+\frac{R_{1}}{T_{1}} e^{-i k s / \varepsilon}\right)+\frac{R_{2}}{T_{2}}\left(\frac{e^{-i k s / \varepsilon}}{\bar{T}_{1}}+\frac{\bar{R}_{1}}{\bar{T}_{1}} e^{i k s / \varepsilon}\right) \\
=\frac{1}{T_{2}}\left(\frac{1}{T_{i}}+\frac{R_{2} \bar{R}_{1}}{\bar{T}_{1}}\right) e^{i k s / \varepsilon}+\frac{1}{T_{2}}\left(\frac{R_{1}}{T_{1}}+\frac{R_{2}}{\bar{T}_{1}}\right) e^{-i k s / \varepsilon} \quad \text { when } s<x .
\end{array}\right.
$$

A comparison of this with (III.6) gives

$$
\frac{1}{T}=\frac{1}{T_{2}}\left(\frac{1}{T_{1}}+\frac{R_{2} \bar{R}_{1}}{\bar{T}_{1}}\right), \quad \frac{R}{T}=\frac{1}{T_{2}}\left(\frac{R_{1}}{T_{1}}+\frac{R_{2}}{T_{2}}\right)
$$

We use formulas (III.13) to calculate $T_{i}, R_{i} \mid i=1,2$ with

$$
\begin{aligned}
& J_{1}=x k, \quad I_{1}=-c k+\int_{x_{2}}^{c}\left(k^{2}-q(y)\right)^{1 / 2} d y, \\
& \Gamma_{1}=\int_{x}^{x_{2}}\left(q(y)-k^{2}\right)^{1 / 2} d y, \\
& J_{2}=c k+\int_{c}^{x_{1}}\left(k^{2}-q(y)\right)^{1 / 2} d y, \quad I_{2}=-(x+p) k, \\
& \Gamma_{2}=\int_{x_{1}}^{x+p}\left(q(y)-k^{2}\right) d y,
\end{aligned}
$$


(III.23)

$$
\begin{aligned}
\frac{1}{T}= & \frac{1}{T_{1} T_{2}}\left(1+\frac{R_{2} \bar{R}_{1} T_{1}}{\bar{T}_{1}}\right) \\
= & \exp \left[-\frac{i k p}{\varepsilon}+\alpha_{1}+\alpha_{2}-i\left(\tau_{1}+\tau_{2}\right)\right] \\
& \times\left\{1+\exp \left[r_{1}+r_{2}+i\left(-\rho_{1}+\rho_{2}+2 \tau_{1}\right)\right]\right\} \\
= & \exp \left[\alpha_{1}+\alpha_{2}+\frac{i k p}{\varepsilon}-\frac{i \Theta}{\varepsilon}+O\left(\varepsilon^{2}\right)\right] \\
& \times\left\{1+\exp \left[r_{1}+r_{2}+\frac{2 i \Theta}{\varepsilon}+O\left(\varepsilon^{2}\right)\right]\right\},
\end{aligned}
$$

where $\Theta=I_{1}+J_{2}$ is defined by (III.17c).

$$
\text { (III.24) } \begin{aligned}
\frac{R}{T}= & \frac{R_{1}}{T_{1} T_{2}}\left(1+\frac{R_{2} T_{1}}{R_{1} \bar{T}_{1}}\right) \\
= & \exp \left[-r_{1}+\alpha_{1}+\alpha_{2}+i\left(\rho_{1}-\tau_{1}-\tau_{2}\right)\right] \\
& \times\left\{1+\exp \left[-r_{2}+r_{1}+i\left(\rho_{2}-\rho_{1}+2 \tau_{1}\right)\right]\right\} \\
\sim & \exp \left[-r_{1}+\alpha_{1}+\alpha_{2}+i\left(\frac{2 J_{1}}{\varepsilon}-\frac{\pi}{2}-\frac{I_{1}+J_{1}+I_{2}+J_{2}}{\varepsilon}+O\left(\varepsilon^{2}\right)\right)\right] \\
& \times\left\{1+\exp \left[-r_{2}+r_{1}+i\left(\frac{2 J_{2}-2 J_{1}+2 I_{1}+2 J_{1}}{\varepsilon}\right)+O\left(\varepsilon^{2}\right)\right]\right\} \\
= & \exp \left[-r_{1}+\alpha_{1}+\alpha_{2}+i\left(\frac{J_{1}-I_{2}}{\varepsilon}-\frac{I_{1}+J_{2}}{\varepsilon}-\frac{\pi}{2}+O\left(\varepsilon^{2}\right)\right)\right] \\
& \times\left\{1+\exp \left[-r_{2}+r_{1}+\frac{2 i}{\varepsilon}\left(I_{1}+J_{2}\right)\right]+O\left(\varepsilon^{2}\right)\right\} \\
= & \exp \left[-r_{1}+\alpha_{1}+\alpha_{2}+i\left(\frac{2 x k+p k}{\varepsilon}-\frac{\Theta}{\varepsilon}-\frac{\pi}{2}\right)+O\left(\varepsilon^{2}\right)\right] \\
& \times\left\{1+\exp \left[r_{1}-r_{2}+\frac{2 i \Theta}{\varepsilon}+O\left(\varepsilon^{2}\right)\right]\right\} .
\end{aligned}
$$

From (III.23) we see

$$
\tau=-\frac{k p}{\varepsilon}+\frac{\Theta}{\varepsilon}-\arg \left[1+e^{r_{1}+r_{2}} \exp \frac{2 i}{\varepsilon} \Theta\right]+O\left(\varepsilon^{2}\right) .
$$

From (III.24) we obtain

(III.26) $\tau-\rho=-\frac{2 x k+p k}{\varepsilon}+\frac{\Theta}{\varepsilon}+\frac{\pi}{2}-\arg \left[1+e^{r_{1}-r_{2}} \exp 2 i \frac{\Theta}{\varepsilon}\right]+O\left(\varepsilon^{2}\right)$.

For $i=1,2$ we have

$$
e^{-r_{i}}=\left(1-e^{-\alpha_{i}}\right)^{1 / 2} \sim 1-\frac{1}{2} e^{-\alpha_{i}}, \quad e^{r_{i}} \sim 1+\frac{1}{2} e^{-\alpha_{i}},
$$

(III.28) $e^{r_{1}+r_{2}} \sim 1+\frac{1}{2}\left(e^{-\alpha_{1}}+e^{-\alpha_{i}}\right), \quad e^{r_{1}-r_{2}} \sim 1+\frac{1}{2}\left(e^{-\alpha_{1}}-e^{-\alpha_{2}}\right)$.

Substituting (III.28) into (III.25)-(III.26) and observing that $\alpha_{i}=L_{i} / \varepsilon$ for $i=1,2$ we obtain formulas (III.16a-b). 
By (III.23) and (III.28) we have

$$
\begin{aligned}
\alpha & =\alpha_{1}+\alpha_{2}+\log \left|1+\frac{R_{2} \bar{R}_{1} T_{1}}{\bar{T}_{1}}\right| \\
& \sim \frac{L_{1}+L_{2}}{\varepsilon}+\log \left|1+\left[1+\frac{e^{-L_{1} / \varepsilon}+e^{-L_{2} / \varepsilon}}{2}\right] e^{2 i \Theta / \varepsilon}\right|
\end{aligned}
$$

This proves (III.16c). Q.E.D.

Our next move is to substitute the expressions obtained for $\tau, \rho, \alpha$ in equation (III.11)-(III.12) and solve for the required eigenvalues. We do this in the original variable $\eta^{2}=1-k^{2}$. We set

$$
\begin{array}{cc}
\quad \theta(\eta)=\Theta(k)=\operatorname{Re} \int_{x}^{x+p}\left(v(y)-\eta^{2}\right)^{1 / 2} d y, \\
\text { (III.31) } \\
\text { (III.32) } \quad l_{1}(\eta, x)=L_{1}(k, x)=\int_{x}^{x_{2}}\left(\eta^{2}-v(y)\right)^{1 / 2} d y \quad \text { (case 2), } \\
\text { (III.33) } \quad l_{2}(\eta, x)=L_{2}(k, x)=\int_{x_{1}}^{x+p}\left(\eta^{2}-v(y)\right)^{1 / 2} d y \quad \text { (case 2), } \\
\text { (III.34) } \zeta(\eta, x)=\int_{x}^{x_{1}}\left(v(y)-\eta_{2}\right)^{1 / 2} d y=\int_{x}^{x_{1}}\left(k^{2}-q(y)\right)^{1 / 2} d y \quad \text { (case 1). }
\end{array}
$$

As $\varepsilon \rightarrow 0$, the quantities which appear in equations (III.11)-(III.12) and were obtained in Lemma III.3 can be written

Case 1. $v(x)>\eta^{2}$.

$$
\begin{gathered}
k p+\tau \sim \frac{\theta(\eta)}{\varepsilon}, \\
\frac{2 k x}{\varepsilon}+\frac{k p}{\varepsilon}+\tau-\rho \sim \frac{\theta(\eta)}{\varepsilon}-\frac{2 \zeta(\eta, x)}{\varepsilon}+\frac{\pi}{2} \\
\alpha \sim \frac{\gamma(\eta)}{\varepsilon} .
\end{gathered}
$$

Case 2. $v(x)<\eta^{2}$.

(III.36a) $k p+\tau \sim \frac{\theta(\eta)}{\varepsilon}-\arg \left\{1+\left[1+\frac{1}{2}\left(e^{-l_{1}(\eta, x) / \varepsilon}+e^{-l_{2}(\eta, x) / \varepsilon}\right)\right] e^{2 i \theta(\eta) / \varepsilon}\right\}$,

(III.36b)

$$
\begin{aligned}
\frac{2 k x}{\varepsilon}+\frac{k p}{\varepsilon}+ & \tau-\rho \\
& \sim \frac{\theta(\eta)}{\varepsilon}+\frac{\pi}{2}-\arg \left\{1+\left[1+\frac{1}{2}\left(e^{-l_{1}(\eta, x) / \varepsilon}-e^{-l_{2}(\eta, x) / \varepsilon}\right)\right] e^{2 i \theta(\eta) / \varepsilon}\right\},
\end{aligned}
$$


(III.36c)

$$
\alpha \sim \frac{1}{\varepsilon}\left(l_{1}(\eta, x)+l_{2}(\eta, x)\right)+\log \left|1+\left[1+\frac{1}{2}\left(e^{l_{1}(\eta, x) / \varepsilon}+e^{-l_{2}(\eta, x) / \varepsilon}\right)\right] e^{2 i \theta(\eta) / \varepsilon}\right| .
$$

The solution of equation (III.11) gives the following result:

THEOREM III.4. Let $\left|\eta_{i}^{2}-\delta_{i}, \eta_{i}^{2}+\delta_{i}\right|$ denote the ith spectral band of the operator $\mathscr{L}_{\varepsilon}(0)$. As $\varepsilon \rightarrow 0$, we let $i \rightarrow \infty$ in such a way that $\eta_{i} \rightarrow \eta$ where $0<\eta<1$. Then

$$
\eta_{i}-\eta_{i+1} \sim \frac{\pi \varepsilon}{\phi(\eta)} \quad \text { where } \phi(\eta)=\left|\frac{d \theta(\eta)}{d \eta}\right|=\operatorname{Re} \int_{x}^{x+p} \frac{\eta}{\left(v(y)-\eta^{2}\right)^{1 / 2}} d y .
$$

$$
\log \delta_{i} \sim-\gamma(\eta) / \varepsilon \quad \text { where } \gamma(\eta) \text { is given by (III.31) }
$$

The calculation is completely straightforward and is omitted. More accurate results can be found in [10].

THEOREM III.5. Let $\eta, \eta_{i}, \delta_{i}$ be as in the previous theorem.

(a) In the region $v(x)>\eta^{2}$, as $\varepsilon \rightarrow 0$ and $i \rightarrow \infty$ in such a way that $\eta_{i} \rightarrow \eta$, the graph of $\nu_{i}(x, 0, \varepsilon)$ is to leading order triangular with oscillation $\sim \pi \varepsilon / \phi(\eta)$ and slopes

$$
\frac{d \nu_{i}}{d x} \sim \frac{2 \eta \zeta_{x}}{\theta_{\eta}-\zeta_{\eta}}<0 \quad \text { and } \quad \frac{d \nu_{i}}{d x} \sim \frac{-2 \eta \zeta_{x}}{\zeta_{\eta}}>0
$$

Furthermore,

(III.40)

$\sum_{k=i-1}^{i}\left(\operatorname{sgn} \frac{\partial \nu_{k}}{\partial x}\right) \varepsilon \log \left|\left[\eta_{i}^{2}-\nu_{k}(x, 0, \varepsilon)\right]+\left[\left(\eta_{i}^{2}-\nu_{k}(x, 0, \varepsilon)\right)^{1 / 2}-\delta_{i}^{2}\right]^{1 / 2}\right| \ll 1$

$$
\text { as } \varepsilon \rightarrow 0 \text {. }
$$

(b) In the region $v(x)<\eta^{2}$ as $\varepsilon \rightarrow 0$ and $i \rightarrow \infty$ in such a way that $\eta_{i} \rightarrow \eta$, we have

$$
\begin{gathered}
\frac{1}{2} \varepsilon \log \left|\eta_{i}^{2}-\nu_{i}(x, 0, \varepsilon)+\left[\left(\eta_{i}^{2}-\nu_{i}(x, 0, \varepsilon)\right)^{2}-\delta_{i}^{2}\right]^{1 / 2}\right| \\
\sim-\frac{\gamma(\eta)}{2}+\left|\int_{\tilde{x}}^{x}\left(\eta^{2}-v(y)\right)^{1 / 2} d y\right| \text { as } \varepsilon \rightarrow 0
\end{gathered}
$$

where $\tilde{x}$ is defined by $l_{1}(\eta, \tilde{x})-l_{2}(\eta, \tilde{x})=0$.

Proof. (a) Region $v(x)>\eta^{2}$. We study equation (III.12) which becomes

(III.42) $\sin \left(\frac{\theta(\eta)}{\varepsilon}+O\left(\varepsilon^{2}\right)\right)=e^{-r} \sin \left[\frac{\theta(\eta)}{\varepsilon}-\frac{2 \zeta(\eta, x)}{\varepsilon}+\frac{\pi}{2}+O\left(\varepsilon^{2}\right)\right]$. 
If, in a first approximation, the exponentially small coefficient $e^{-r}$ is dropped we obtain the solutions

$$
\begin{gathered}
\frac{\theta(\eta)}{\varepsilon}-\frac{\zeta(\eta, x)}{\varepsilon}=-\frac{\pi}{4}+m \pi+O\left(\varepsilon^{2}\right), \\
\frac{\zeta(\eta, x)}{\varepsilon}=-\frac{\pi}{4}+m^{\prime} \pi+O\left(\varepsilon^{2}\right),
\end{gathered}
$$

where $m$ and $m^{\prime}$ are integers. We solve these asymptotically as $\varepsilon \rightarrow 0$. We set $\eta=\eta_{0}+\varepsilon \eta_{1}+\cdots, x=x_{0}+\varepsilon x_{1}+\cdots$. Thus,

$$
\begin{aligned}
& \theta(\eta)=\theta\left(\eta_{0}\right)+\varepsilon \theta_{\eta}\left(\eta_{0}\right) \eta_{1}+\cdots, \\
& \zeta(\eta, x)=\zeta\left(\eta_{0}, x_{0}\right)+\zeta_{\eta}\left(\eta_{0}, x_{0}\right) \eta_{1}+\varepsilon \zeta_{x}\left(\eta_{0}, x_{0}\right) x_{1}+\cdots .
\end{aligned}
$$

Let

$$
\begin{aligned}
\theta\left(\eta_{0}\right)-\zeta\left(\eta_{0}, x_{0}\right) & =m_{0} \pi+A\left(\eta_{0}, x_{0}\right), & & |A| \leqslant \pi, \\
\zeta\left(\eta_{0}, x_{0}\right) & =m_{0}^{\prime} \pi+B\left(\eta_{0}, x_{0}\right), & & |B| \leqslant \pi,
\end{aligned}
$$

where $m_{0}, m_{0}^{\prime}$ are integers. We obtain easily

$$
\begin{array}{r}
{\left[\theta_{\eta}\left(\eta_{0}\right)-\zeta_{\eta}\left(\eta_{0}, x_{0}\right)\right] \eta_{1}-\zeta_{x}\left(\eta_{0}, x_{0}\right) x_{1}=-\pi / 4+m_{1} \pi+A\left(\eta_{0}, x_{0}\right),} \\
\zeta_{\eta}\left(\eta_{0}, x_{0}\right) \eta_{1}-\zeta_{x}\left(\eta_{0}, x_{0}\right) x_{1}=-\pi / 4+m_{1}^{\prime} \pi+B\left(\eta_{0}, x_{0}\right),
\end{array}
$$

where $m_{1}, m_{1}^{\prime}$ are integers. When $\varepsilon$ is small, the dependence of $\eta_{i}=\nu_{i}^{1 / 2}(x, 0, \varepsilon)$ on $x$ is given by a triangular function as in Figure 3.1.

Our analysis is not accurate enough at the vertices of the triangles and hence inadequate to justify (III.40). For example, we know that the graphs of $\nu_{i}$ and $\nu_{i+1}$ do not intersect but are separated by an exponentially small band (see Figure 3.1.2). We can correct our result near the vertices by

(i) Not dropping the factor $e^{-r}$ from (III.42).

(ii) Observing that in this region

$1-\left|\sin \left(\frac{\theta(\eta)}{\varepsilon}+O\left(\varepsilon^{2}\right)\right)\right| \ll 1, \quad 1-\left|\sin \left(\frac{\theta(\eta)}{\varepsilon}-\frac{2 \zeta(\eta, x)}{\varepsilon}+\frac{\pi}{2}+O\left(\varepsilon^{2}\right)\right)\right| \ll 1$,

and using the formula

$$
\sin (\pi / 2-\lambda) \sim\left(1-\lambda^{2}\right)^{1 / 2} \quad(\lambda \rightarrow 0) .
$$

This reduces (III.42) to a quadratic equation. The $i$ th band is obtained as the set of $\eta^{2}$ 's for which the discriminant of the quadratic equation is negative. Relation (III.40) is verified easily. The calculation is straightforward and is omitted.

(b) Region $v(x)<\eta^{2}$. Necessarily, if $\eta(x, \varepsilon)$ is a solution of (III.12) we have

$$
\theta(\eta) / \varepsilon \equiv \pi / 2+o(1) \quad(\bmod \pi)
$$

otherwise in equations (III.36a-b) $\arg \{\cdot\} \sim \theta(\eta) / \varepsilon$ and

$$
k p+\tau \sim \frac{\theta(\eta)}{\varepsilon}-\frac{\theta(\eta)}{\varepsilon}=0, \quad \frac{2 k x}{\varepsilon}+\frac{k p}{\varepsilon}+\tau-\rho \sim \frac{\theta(\eta)}{\varepsilon}-\frac{\theta(\eta)}{\varepsilon}+\frac{\pi}{2} \sim \frac{\pi}{2} .
$$

Then equation (III.12) would lead to the contradiction $0=1$. The solutions of equation (III.12) are essentially the Dirichlet eigenvalues $\nu_{i}(x, 0, \varepsilon)$. Thus equation 
(III.46) implies that the total variation of each $\nu_{i}(x, 0, \varepsilon)$ is of order $o(\varepsilon)$ in the region $v(x)<\nu_{i}^{2}$. There is a point $\tilde{x}$ for which $l_{1}(\eta, \tilde{x})=l_{2}(\eta, \tilde{x})$. At this point equation (III.36b) becomes

$$
\frac{2 k x}{\varepsilon}+\frac{k p}{\varepsilon}+\tau-\rho \sim \frac{\theta(\eta)}{\varepsilon}+\frac{\pi}{2}-\frac{\theta(\eta)}{\varepsilon} \sim \frac{\pi}{2} .
$$

Equation (III.12) becomes $\sin (k p / \varepsilon+\tau) \sim 1$. By Theorem III.1 $\nu_{i}(x, 0, \varepsilon)$ is then $o(\varepsilon)$ close to one of the two end points of the $i$ th gap. It is easy to check in this theorem that

$$
\sin (k p / \varepsilon+\tau)=+e^{-r} \Rightarrow \nu_{i}(x, 0, \varepsilon)=\xi_{2 i-1} .
$$

By our previous remark

$$
\nu_{i}(x, 0, \varepsilon)-\xi_{2 i-1}=o(\varepsilon)
$$

will be valid as long as $\eta^{2}>v(x)$ is satisfied. We will not proceed with the complete solution of (III.12) to obtain this difference. Instead, we use the crude result of equation (III.48) and the previous remark to derive the solution from equations (1.3):

$$
\left|\frac{d \nu_{i}}{d x / \varepsilon}\right| \sim\left|\frac{2 \prod_{k=1}^{N}\left[\left(\nu_{i}-\xi_{2 k-1}\right)\left(\nu_{i}-\xi_{2 k}\right)\right]^{1 / 2}}{\prod_{k=0 ; k \neq i}^{N}\left(\nu_{i}-\nu_{k}\right)}\right| .
$$

We replace $\xi_{2 k}$ when $k \neq i-1$ with $\eta_{k+1}^{2}$ and we replace $\xi_{2 k-1}$ when $k \neq i$ with $\eta_{k}^{2}$

$$
\begin{gathered}
\left|\frac{d \nu_{i}}{d x / \varepsilon}\right| \sim 2\left|\left(\nu_{i}-\xi_{2 i-1}\right)\left(\nu_{i}-\xi_{2 i-2}\right)\right|^{1 / 2}\left\{\prod_{\substack{v_{k}>v(x) \\
k \neq i}}\left|\frac{\nu_{i}-\eta_{k}^{2}}{\nu_{i}-\nu_{k}}\right|\right\}\left(\nu_{i}-v(x)\right)^{1 / 2} \\
\cdot \prod_{\nu_{k}<v(x)} \frac{\left|\left(\nu_{i}-\eta_{k}^{2}\right)\left(\nu_{i}-\eta_{k+1}^{2}\right)\right|^{1 / 2}}{\left|\nu_{i}-\nu_{k}\right|} .
\end{gathered}
$$

Claim. The products over $\nu_{k}>v(x)$ and $\nu_{k}<v(x)$ are equal to 1 to leading order.

Proof of Claim. (i) The product over $\nu_{k}>v(x)$ can be replaced by 1 because we showed that $\left|\nu_{k}-\xi_{2 k-1}\right|$ and hence $\left|\nu_{k}-\eta_{k}^{2}\right|$ is of order $o(\varepsilon)$.

$$
\begin{aligned}
\prod_{\nu_{k}<v(x)}\left|\frac{\left(\nu_{i}-\eta_{k}^{2}\right)\left(\nu_{i}-\eta_{k+1}^{2}\right)}{\left(\nu_{i}-\nu_{k}\right)^{2}}\right|^{1 / 2} & =\prod_{\nu_{k}<v(x)}\left|\left(1+\frac{\nu_{k}-\eta_{k}^{2}}{\nu_{i}-\nu_{k}}\right)\left(1+\frac{\nu_{k}-\eta_{k+1}^{2}}{\nu_{i}-\nu_{k}}\right)\right|^{1 / 2} \\
& \sim \prod_{\nu_{k}<v(x)}\left|1+\frac{2\left[\nu_{k}-\frac{1}{2}\left(\eta_{k}^{2}+\eta_{k+1}^{2}\right)\right]}{\nu_{i}-\nu_{k}}\right|
\end{aligned}
$$

It is clear that for $\nu_{k}<v(x)$ :

$$
\sum_{\eta^{2}<\nu_{k}<\eta^{2}+\delta_{\varepsilon}}\left\{\nu_{k}-\frac{1}{2}\left(\eta_{k}^{2}+\eta_{k+1}^{2}\right)\right\} \ll \delta_{\varepsilon} \quad \text { when } \varepsilon \ll \delta_{\varepsilon} \ll 1 .
$$

Thus the product over $\nu_{k}<v(x)$ can be replaced by 1 and the claim is proven. 
Equation (III.50) becomes

(III.52)

$$
\frac{\operatorname{sgn} \partial \nu_{i} / \partial x}{\left|\left(\nu_{i}-\xi_{2 i-1}\right)\left(\nu_{i}-\xi_{2 i-2}\right)\right|^{1 / 2}} d \nu_{i} \sim \frac{2}{\varepsilon}\left(\nu_{i}-v(x)\right)^{1 / 2} d x-\frac{2}{\varepsilon}\left(\eta_{i}^{2}-v(x)\right)^{1 / 2} d x
$$

We recall that $\xi_{2 i-1}=\eta_{i}^{2}-\delta_{i}, \xi_{2 i-2}=\eta_{i}^{2}+\delta_{i}$. We substitute in (III.52) and integrate

(III.53) $\log \left|\frac{\left(\eta_{i}^{2}-\nu_{i}\right)+\left[\left(\eta_{i}^{2}-\nu_{i}\right)^{2}-\delta_{i}^{2}\right]^{1 / 2}}{\delta_{i}}\right| \sim \frac{2}{\varepsilon}\left|\int_{\tilde{x}}^{x}\left(\eta^{2}-v(y)\right)^{1 / 2} d y\right|$,

where $\tilde{x}$ is the point at which $l_{1}=l_{2}$. At this point $\nu_{i}=\xi_{2 i-1}=\eta_{i}^{2}-\delta_{i}$. Thus, at $\tilde{x}$ both sides of (III.53) are equal to zero.

Finally by using (III.38) we obtain

$$
\begin{aligned}
\log \mid \eta_{i}^{2}- & \nu_{i}(x, 0, \varepsilon)+\left[\left(\eta_{i}^{2} \nu_{i}(x, 0, \varepsilon)\right)^{2}-\delta_{i}^{2}\right]^{1 / 2} \mid \\
& \sim-\frac{\gamma(\eta)}{\varepsilon}+\left|\frac{2}{\varepsilon} \int_{\tilde{x}}^{x}\left(\eta^{2}-v(y)\right)^{1 / 2} d y\right| . \quad \text { Q.E.D. }
\end{aligned}
$$

THeOREM III.6. Let $j(\varepsilon)$ be such that $\eta_{j} \rightarrow \eta \in(0,1)$ as $\varepsilon \rightarrow 0$. We define (III.55)

$$
r(\eta, x, t)=\lim _{\varepsilon \rightarrow 0} r_{j}(x, t, \varepsilon),
$$

where $r_{j}(x, t, \varepsilon)$ is given by (3.13). Let $x_{ \pm}(\eta)$ be defined by Figure 5.1. Then

(a) When $x_{-}(\eta)<x<x_{+}(\eta)$ we have

$$
r(\eta, x, 0)=0
$$

(b) When $x_{+}(\eta)<x<x_{-}(\eta)+p$ we have

$$
r(\eta, x, 0)= \begin{cases}\int_{x_{+}}^{x}\left(\eta^{2}-v(y)\right)^{1 / 2} d y & \text { when } x<\tilde{x}, \\ -\int_{x}^{x_{-}+p}\left(\eta^{2}-v(y)\right)^{1 / 2} d y & \text { when } x>\tilde{x}\end{cases}
$$

where $\tilde{x}$ is defined by the equation

$$
\int_{x_{+}}^{\tilde{x}}\left(\eta^{2}-v(y)\right)^{1 / 2} d y=\frac{1}{2} \gamma(\eta)
$$

Proof. Relation (III.56) follows from (III.40). We now prove (III.57). By (3.13) and (III.41)

$$
r_{j}(x, 0, \varepsilon) \sim\left[\frac{1}{2} \gamma(\eta)-\left|\int_{\tilde{x}}^{x}\left(\eta^{2}-v(y)\right)^{1 / 2} d y\right|\right] \sigma_{j} .
$$

Case $x>\tilde{x}$.

(III.60)

$r_{j}(x, 0, \varepsilon) \sim\left[\frac{1}{2} \gamma(\eta)-\int_{\tilde{x}}^{x_{-}+p}\left(\eta^{2}-v(y)\right)^{1 / 2} d y+\int_{x}^{x_{-}+p}\left(\eta^{2}-v(y)\right)^{1 / 2} d y\right] \sigma_{j}$. 
By the definition of $\tilde{x}$ we have

$$
\int_{\tilde{x}}^{x_{-}+p}\left(\eta^{2}-v(y)\right)^{1 / 2} d y=\frac{1}{2} \gamma(\eta) .
$$

From (III.41) when $x>\tilde{x}$ we have

$$
\frac{\partial \nu_{j}}{\partial x}=\sigma_{j}=-1
$$

Therefore

$$
r_{j}(x, 0, \varepsilon) \sim-\int_{x}^{x_{-}+p}\left(\eta^{2}-v(y)\right)^{1 / 2} d y \quad \text { as } \varepsilon \rightarrow 0 .
$$

Case $x<\tilde{x}$.

$$
\begin{aligned}
r_{j}(x, 0, \varepsilon) & \sim\left[\frac{1}{2} \gamma(\eta)+\int_{\tilde{x}}^{x_{-}+p}\left(\eta^{2}-v(y)\right)^{1 / 2} d y-\int_{x}^{x_{-}+p}\left(\eta^{2}-v(y)\right)^{1 / 2} d y\right] \sigma_{j} \\
& =\gamma(\eta)-\int_{x}^{x_{-}+p}\left(\eta^{2}-v(y)\right)^{1 / 2} d y=\int_{x_{+}}^{x}\left(\eta^{2}-v(y)\right)^{1 / 2} d y \text {. Q.E.D. }
\end{aligned}
$$

Acknowledgments. I thank Peter Lax, Joe Keller, Dave McLaughlin, George Papanicolaou, Henry McKean, Dave Levermore, and Greg Forest for many discussions from which this study has greatly benefited. I also thank the referee for many excellent suggestions.

\section{REFERENCES}

1. C. M. Bender and S. A. Orszag, Advanced mathematical methods for scientists and engineers, McGraw-Hill, New York, 1978.

2. E. Date and S. Tanaka, Periodic multisolitons of the Korteweg-de Vries equation and Toda lattice, Progr. Theoret. Phys. Suppl., no. 59, 1976.

3. B. A. Dubrovin, V. B. Matveev, and S. P. Novikov, Nonlinear equations of Korteweg-de Vries type, finite zoned linear operators, and Abelian varieties, Uspekhi Mat. Nauk 31 (1976), 55-136.

4. H. Flaschka, M. G. Forest, and D. W. McLaughlin, Multiphase averaging and the inverse spectral solution of the Korteweg-de Vries equation, Comm. Pure Appl. Math. 33 (1980), 739-784.

5. J. B. Keller, Discriminant, transmission coefficient and stability bands of Hill's equation, J. Math. Phys. 25 (1984), 2903-2904.

6. P. D. Lax and C. D. Levermore, The small dispersion limit of the Korteweg-de Vries equation. I, II, III, Comm. Pure Appl. Math. 36 (1983), 253-290, 571-593, 809-829.

7. H. P. McKean and E. Trubowitz, Hill's operator and hyperelliptic function theory in the presence of infinitely many branch points, Comm. Pure Appl. Math. 29 (1976), 146-226.

8. H. P. McKean and P. vanMoerbeke, The spectrum of Hill's equation, Invent. Math. 30 (1975), 217-274.

9. E. Trubowitz, The inverse problem for periodic potentials, Comm. Pure Appl. Math. 30 (1977), 321-337.

10. M. I. Weinstein and J. B. Keller, Asymptotic behavior of stability regions for Hill's equation (to appear).

11. S. Venakides, The zero dispersion limit of the Korteweg-de Vries equation with nontrivial reflection coefficient, Comm. Pure Appl. Math. 38 (1985), 125-155.

12. , The generation of modulated wavetrains in the solution of the Korteweg-de Vries equation, Comm. Pure Appl. Math. 38 (1985), 883-909.

13. G. B. Whitham, Linear and nonlinear waves, Wiley, New York, 1974. 
14. D. W. McLaughlin, On the construction of modulating multiphase wavetrains, Preprint, University of Arizona, 1982.

15. M. G. Forest and D. W. McLaughlin, Modulation of sinh-Goordon and sine-Gordon wavetrains, Stud. Appl. Math. 68 (1983), 11-59.

16. __ Modulation of perturbed KdV wavetrains, SIAM J. Appl. Math. 44 (1984), 287-300.

17. N. Ercolani, M. G. Forest, and D. W. McLaughlin, Modulational stability of two phase sine-Gordon wavetrains, Stud. Appl. Math. 71 (1984).

18. M. E. Schonbek, Convergence of solutions to nonlinear dispersive equations, Comm. Partial Differential Equations 7 (1982), 959-1000.

19. R. J. DiPerna, Measured valued solutions to conservation laws, Arch. Rational Mech. Anal. (1985), 223-270.

20. L. Tartar, Compensated compactness and applications to partial differential equations, Research Notes in Math., Nonlinear Anal. and Mech. Heriot-Watt Symposium, Vol. 4 (R. J. Knops, ed.), Pitman Press, New York, 1979.

21. C. D. Levermore, The small dispersion limit of the Korteweg-de Vries equation: Multiwell initial data, preprint.

22. S. Venakides, The small dispersion limit of the Korteweg-de Vries equation, Proc. Conf. P. D. Lax's 60th birthday, MSRI (A. Chorin and A. Majda, eds.), Springer, 1987.

Department of Mathematics, Stanford University, Stanford, California 94305

Current address: Department of Mathematics, Duke University, Durham, North Carolina 27706 